ARTICLE

https://doi.org/10.1038/s41467-021-22230-z

\title{
The survival and function of IL-10-producing regulatory $B$ cells are negatively controlled by SLAMF5
}

Lihi Radomir ${ }^{1}$, Matthias P. Kramer (10 1, Michal Perpinial ${ }^{1}$, Nofar Schottlender $^{1}$, Stav Rabani ${ }^{1}$, Keren David (10 1, Anna Wiener ${ }^{1}$, Hadas Lewinsky ${ }^{1}$, Shirly Becker-Herman ${ }^{1}$, Rina Aharoni ${ }^{1}$, Ron Milo ${ }^{2,3}$, Claudia Mauri $^{4}$ \& Idit Shachar (1D ${ }^{1 \times}$

B cells have essential functions in multiple sclerosis and in its mouse model, experimental autoimmune encephalomyelitis, both as drivers and suppressors of the disease. The suppressive effects are driven by a regulatory B cell (Breg) population that functions, primarily but not exclusively, via the production of IL-10. However, the mechanisms modulating IL-10producing Breg abundance are poorly understood. Here we identify SLAMF5 for controlling $\mathrm{IL}-10^{+}$Breg maintenance and function. In EAE, the deficiency of SLAMF5 in B cells causes accumulation of $\mathrm{IL} 10^{+}$Bregs in the central nervous system and periphery. Blocking SLAMF5 in vitro induces both human and mouse IL-10-producing Breg cells and increases their survival with a concomitant increase of a transcription factor, c-Maf. Finally, in vivo SLAMF5 blocking in EAE elevates IL-10+ Breg levels and ameliorates disease severity. Our results suggest that SLAMF5 is a negative moderator of $\mathrm{IL}-10^{+}$Breg cells, and may serve as a therapeutic target in MS and other autoimmune diseases.

\footnotetext{
${ }^{1}$ Department of Immunology, The Weizmann Institute of Science, Rehovot, Israel. ${ }^{2}$ Department of Neurology, Barzilai University Medical Center, Ashkelon, Israel. ${ }^{3}$ Faculty of Health Sciences, Ben-Gurion University of the Negev, Beer-Sheva, Israel. ${ }^{4}$ Centre for Rheumatology Research, Department of Medicine, University College London, London, UK. ${ }^{凶}$ email: idit.shachar@weizmann.ac.il
} 
$\mathrm{M}$ ultiple sclerosis (MS) is an autoimmune disease of the central nervous system (CNS) leading to chronic neurological disabilities. It is characterized by extensive inflammation and demyelination, accompanied by axonal and neuronal damage. The disease is heterogeneous in its clinical manifestation and progression, as well as in its pathological mechanisms ${ }^{1}$.

MS has been considered a primarily T-cell-mediated disease in which $\mathrm{T}$ helper (Th)-1 and Th17 effector cells react against myelin components of the CNS, initiating a vicious inflammatory cascade in the CNS. In recent years, it was demonstrated that B cells are also essential for MS progression. B-cells activate and support the T-cell response through secretion of proinflammatory cytokines and were suggested to act as antigenpresenting cells $(\mathrm{APC})^{2}$. In addition, autoantibodies against elements of the CNS are found in the cerebrospinal fluid (CSF) of MS patients $\mathrm{s}^{3,4}$ and serve as a biomarker for the disease $\mathrm{e}^{5}$. These immunoglobulins (Ig) were shown to localize within lesions and near damaged myelin and were demonstrated, ex vivo and in vitro, to cause axonal damage by inducing complementmediated tissue injury ${ }^{3,6}$.

Essential data on MS have been obtained using the animal model, experimental autoimmune encephalomyelitis (EAE), induced by immunization of mice with myelin-derived antigens, such as myelin oligodendrocyte glycoprotein $(\mathrm{MOG})^{7,8}$. MOGinduced EAE models are divided into B-cell-dependent disease, induced by the recombinant protein $\mathrm{MOG}_{1-117}$ (rMOG), and Bcell-independent disease, induced by the peptide $\mathrm{MOG}_{35-55}{ }^{9}$. In the rMOG model, B cells are activated by MOG and act as antibody-secreting cells and APCs. B-cell depletion in this model mitigates the disease and reduces effector T-cell activity9. B cells in the $\mathrm{MOG}_{35-55}$ model are not activated by this MOG form and are not required for disease development, and their depletion leads to clinical exacerbation. The pathogenesis of this model is mediated mainly by $\mathrm{CD}^{+}$effector $\mathrm{T}$ cells ${ }^{9-11}$, while Treg and Th2 responses may offer protection from the disease $e^{12-14}$.

In addition to the detrimental role of B cells in MS, B cells are also essential in disease mitigation ${ }^{15}$ and restriction of inflammation. This is achieved by regulatory B cells (Bregs), a subpopulation of $\mathrm{B}$ cells that is functionally characterized by its ability to suppress the immune response. Bregs were shown, both in humans and in mice, to maintain immune tolerance via diverse regulatory mechanisms, including the secretion of the antiinflammatory cytokine, IL-10 IL-35, TGF- $\beta$, and by the expression of FasL, GITRL, PD-1, and CD7316,17. Mice lacking B cells, due to their genetic background or B-cell-depleting treatments ${ }^{18-20}$, could not recover from the disease, while the transfer of IL-10-producing B cells suppressed the inflammation and reduced disease severity 21,22 .

In humans and mice, Bregs comprise a group of B-cell subpopulations identified by specific markers. Even though several transcription factors (TFs) were found to be important for Bregs, none of these were found to be expressed by all the Breg populations or to act as a common identifying marker. Among their various functions, Bregs maintain the balance between Tregs and Th1/Th17 populations; in addition, they prevent differentiation of naive $\mathrm{T}$ cells into Th1 and Th17 while inducing Treg differentiation and cell expansion, which further suppresses pathological Th1 and Th17 responses ${ }^{23,24}$.

Cross-talk of B cells with their environment is crucial for their survival, activation, and differentiation. The signaling lymphocytic activating molecules (SLAM) family of immunomodulating receptors helps mediate the interaction of immune cells with their microenvironment ${ }^{25}$. This family, which consists of nine members, is differentially expressed mainly on immune cells. In most SLAM members, the extracellular segment is composed of two Ig- like domains, and they contain multiple immunoreceptor tyrosine-based switch motifs (ITSMs) on their cytoplasmic tail.

SLAMF5 (CD84), a member of the SLAM family, is a selfligand receptor that forms homophilic dimers ${ }^{26-28}$. During cellto-cell interactions, it acts both as an adhesion and signaling molecule $^{29}$. Following its activation, SLAMF5 recruits adaptors of the SLAM adaptor protein (SAP) family which induces downstream signaling. SLAMF5 was previously reported to mediate the interactions between chronic lymphocytic leukemia (CLL) cells and their microenvironment ${ }^{30}$, leading to the support of CLL survival ${ }^{31}$ and to the suppression of T-cell activity via PD-L1 ${ }^{32}$. Although SLAMF5 has an essential role in the regulation of the immune response, its direct role in autoimmunity was yet to be examined.

In this study, we investigate the role of SLAMF5 in EAE. We show that abrogating SLAMF5 interactions, by genetic deficiency or blocking antibody, alleviates disease in MOG-induced EAE mice while inducing the accumulation of IL-10-expressing Bregs. In vitro blocking of SLAMF5 expressed on B cells leads to a specific increase of Breg viability and induces expression of the IL-10-regulating transcription factor, c-MAF. These results support the role of the SLAMF5 receptor in modulating the accumulation of IL-10-producing Bregs during autoimmunity.

\section{Results}

SLAMF5 enhances disease severity in EAE. To examine the effect of SLAMF5 in EAE, we injected wild-type (WT) and SLAMF5-deficient $\left(\right.$ slamf $\left.^{-1-}\right)$ mice with $\mathrm{MOG}_{35-55}$ peptide in order to induce EAE. We monitored disease progression over 26 days using the EAE scoring system and changes in body weight.

The clinical impairment of WT mice typically appeared 10 days after disease induction, increasing in severity and reaching a mean clinical score of $3.76 \pm 0.2$ by day 18 , at which point disease incidence was $100 \%$. In contrast, slamf5 $5^{-/-}$mice exhibited delayed disease onset and milder motor dysfunction with a maximal mean score of $2.1 \pm 0.25$ at day 18 , and incidence of only 78\% (Fig. 1a and Supplementary Fig. 1a). Differences between WT and slamf $5^{-1-}$ mice were already evident from day 12 and persisted until the end of the experiment. Furthermore, the difference was found significant according to the area under the curve graphing disease severity (AUC, inset in Fig. 1a). The amelioration of disease was accompanied by reduced weight loss in the slamf5 $5^{-1-}$ compared to the WT group (Fig. 1b, AUC in inset), together indicating that slamf $5^{-I}$ mice are partially protected from EAE.

Since Th1 and Th17 effector cells promote EAE progression, and recovery is promoted by Treg cells, we analyzed these populations. During the active phase of the disease (day 15), we found no differences in the Th1 (Tbet and effector cytokine IFN $\gamma$ ) or the Th17 population (ROR $\gamma \mathrm{T}$ and effector cytokine IL-17) in the spleen or lymph nodes (Fig. 1c, d). Furthermore, no differences were found in the Treg (FOXP $3^{+} \mathrm{CD} 25^{+}$, Fig. 1c, d), total CD4 (Supplementary Fig. 1c), or Th2 (Gata3 and cytokine IL-4, Supplementary Fig. 1d, e) populations. Examination of the cytokine $\mathrm{TNF} \alpha$, which supports inflammation in $\mathrm{EAE}^{33}$, also showed no differences between WT and slamf5 ${ }^{-1-}$ (Supplementary Fig. 1f).

The main site of inflammation in the MOG-induced EAE model is the spinal cord (SC) ${ }^{34}$. Due to the low number of T cells in the SC, we pooled the SCs of four to five mice to perform staining for effector cytokines. Interestingly, the percent and numbers of $\mathrm{CD}^{+} \mathrm{IL}-17^{+}$(Fig. 1e-g) and $\mathrm{CD} 4^{+} \mathrm{IFN} \gamma^{+} \mathrm{T}$ cells (Fig. $1 \mathrm{~h}-\mathrm{j}$ ) were reduced in slamf5 $5^{-1-}$ mice, accompanied by an increase of $\mathrm{CD} 4^{+} \mathrm{IL}-10^{+} \mathrm{T}$ cells (Fig. $\left.1 \mathrm{k}-\mathrm{m}\right)$. 


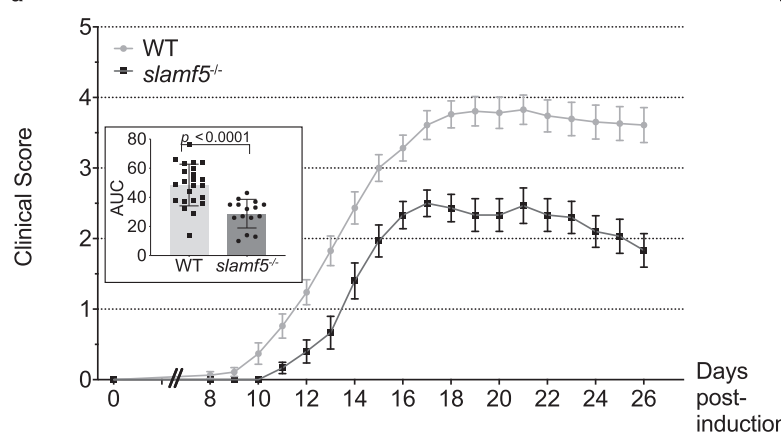

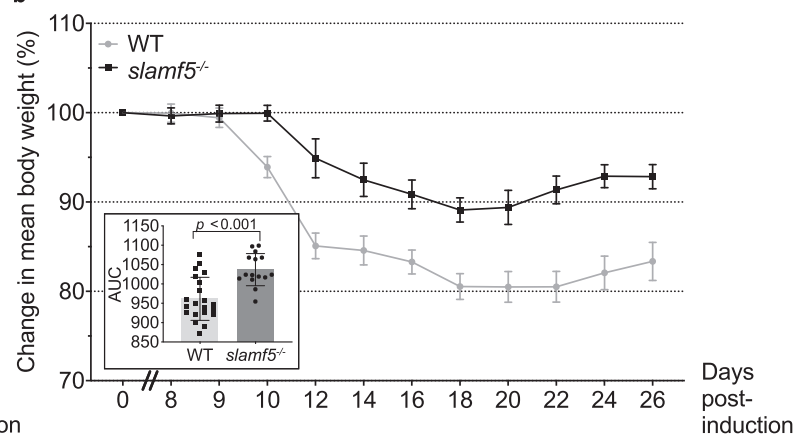

d Lymph Nodes - CD4 ${ }^{+} \mathrm{CD} 3^{+}$

c Spleen - CD4 $4^{+} \mathrm{CD} 3^{+}$

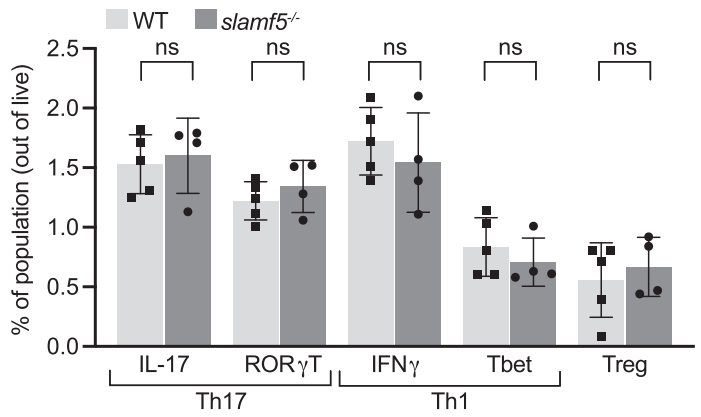

e Spinal cord - CD4 $4^{+} \mathrm{IL}-17^{+}$

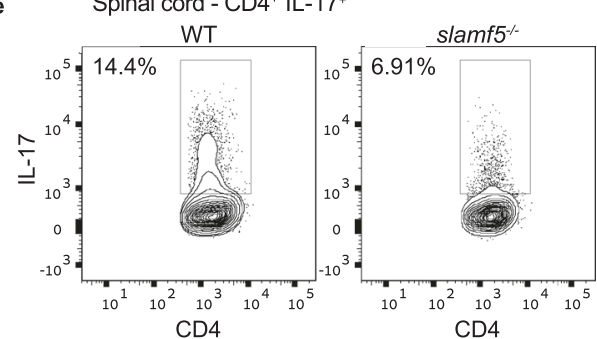

CD4

CD4

h Spinal cord - CD4 $4^{+}$IFN $\gamma^{+}$

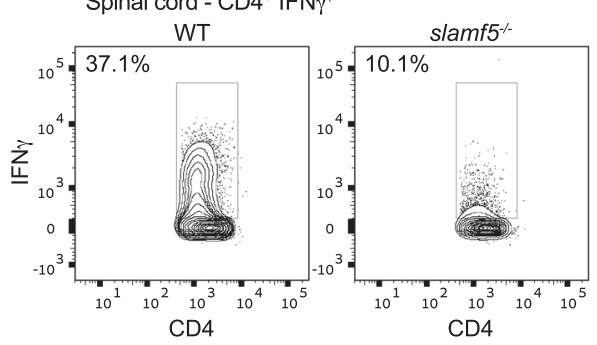

$\mathbf{k}$

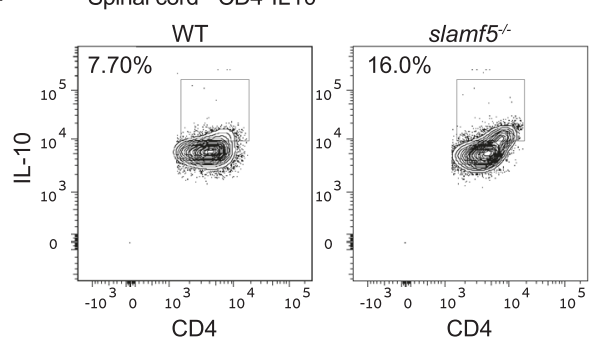

This downregulation of effector T cells in the SC may be due to a disruption in antigen presentation by microglia, monocytes (perivascular macrophages and dendritic cells), and/or B cells residing in the $\mathrm{SC}^{35-37}$. However, no differences were found in the levels of MHC class II expression on these populations (Supplementary Fig. 1h, i), indicating that antigen presentation is
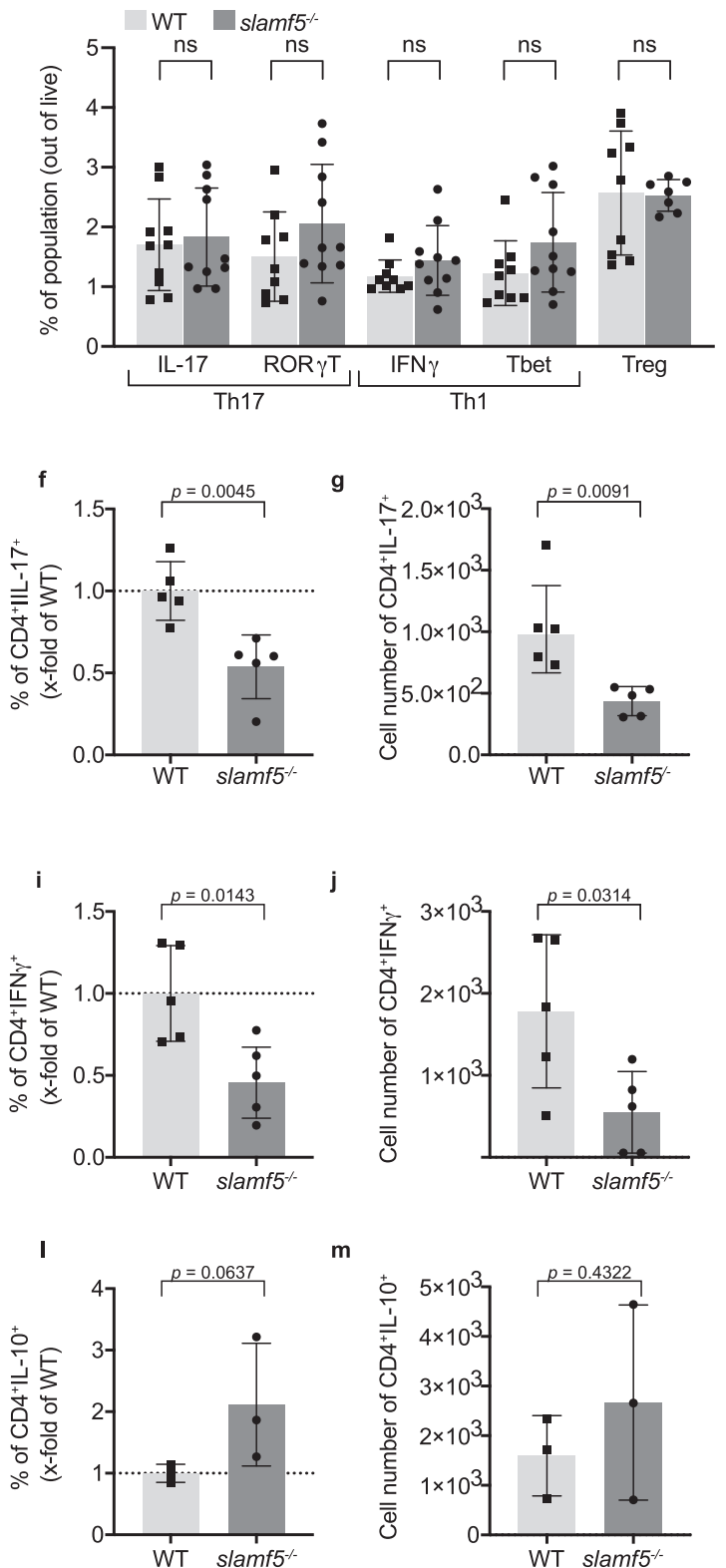

m

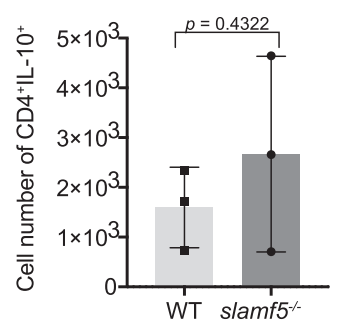

intact, and the effect of SLAM5 deficiency must be due to another mechanism.

SLAMF5 negatively controls $\mathrm{IL}^{-10^{+}}$Breg accumulation. To further investigate the downregulation of effector $\mathrm{T}$ cells in the 
Fig. 1 SLAMF5 deficiency mitigates EAE and increases Breg levels. EAE (MOG $35-55)$ was induced in WT and slamf5-/- mice. Mice were followed for 26 days. a) Daily mean clinical scoring of the disease (WT group $n=23$; slamf5 $5^{-/}$group $n=15$, two representative independent experiments out of seven similar experiments). b Change in mean body weight shown as percent of initial body weight (WT group $n=21$; slamf5-/- group $n=15$, two representative independent experiments out of seven similar experiments). c, d On day 15, spleens, lymph nodes (LNs), and spinal cords were collected, leukocytes were incubated overnight with $\mathrm{MOG}_{35-55}$ and analyzed by FACS for Th1 T cells (IL-17+ and ROR $\gamma \mathrm{T}^{+}$), Th17 T cells (IFN $\gamma^{+}$and T-bet ${ }^{+}$), and Treg (CD25+ $\mathrm{FOXP3}^{+}$) in (c) spleen (WT $n=5$; slamf5 ${ }^{-/-} n=4$, one experiment) (d) LNs, with gating for CD3 ${ }^{+} \mathrm{CD}^{+}$. (WT $n=9-10$; slamf5-/- $n=7-10$, two independent experiments). e-m Four to five spinal cords were pooled together and analyzed for cytokine expression; showing representative histograms, percentage of secreting population, and cell count for: $\mathbf{e}-\mathbf{g} \mathrm{IL}-17^{+}\left(\mathrm{WT} n=5\right.$; slamf5 ${ }^{-/-} n=5$, one experiment), $\mathbf{h}-\mathbf{j} \mathrm{IFN} \gamma^{+} \mathrm{T}\left(\mathrm{WT} n=5\right.$; slamf5 ${ }^{-/-} n=4$, one experiment), and $\mathbf{k}-\mathbf{m} \mathrm{IL}-10^{+}\left(\mathrm{WT} n=3\right.$; slamf5 ${ }^{-/}-n=3$, one experiment) under the gate of $\mathrm{CD} 45^{+} \mathrm{CD}_{11 \mathrm{~b}}{ }^{-} \mathrm{CD} 3^{+} \mathrm{CD} 4+$. Data expressed as mean $\pm \mathrm{s}$. e.m. (a, b) and mean \pm s.d. (a, $\mathbf{b}$ insets, $\mathbf{c}, \mathbf{d}, \mathbf{f}, \mathbf{g}, \mathbf{i}, \mathbf{j}, \mathbf{I}, \mathbf{m})$. Mann-Whitney test (a, $\mathbf{b}$ insets). Unpaired Student's $t$ test with $95 \%$ confidence levels two-tailed $(\mathbf{c}, \mathbf{d}, \mathbf{f}, \mathbf{g}, \mathbf{i}, \mathbf{j}, \mathbf{m})$, one-tailed (I). ( $\left.{ }^{\star \star \star} P<0.001,{ }^{\star \star \star \star} P<0.0001\right)$. Gating strategy for $\mathbf{c}$ and $\mathbf{d}$ is shown in Supplementary Fig. 1a(I.-IV.), gating strategy for $\mathbf{e}-\mathbf{m}$ is shown in Supplementary Fig. 1g.

SC, we analyzed the population of B cells that exert regulatory function, suppress the $\mathrm{CD} 4^{+}$effector response, and produce IL10 (IL-10+ $0^{+}$regulatory B cells; IL- $10^{+}$Bregs or Bregs) ${ }^{38}$. Due to the low number of B cells in the SC, the SCs of four to five mice with matching scores were combined to perform IL-10 staining. To our surprise, we found a significant increase in Breg percentage in the SC of the slamf $5^{-1-}$ mice (Fig. 2a, b).

To determine whether the Breg population is affected only in the SC, we next analyzed Bregs (CD19+ $\left.\mathrm{IL}-10^{+}\right)$in the lymphoid organs. We observed a significant increase in the numbers of Bregs on day 15, both in the spleen and LN (Fig. 2c, d). This increase was limited to the IL-10-expressing populations and was not detected in the IL- $10^{\text {neg }}$ total $\mathrm{B}$ cells or in the $\mathrm{B}$ subpopulation; the mature, marginal zone $(\mathrm{Mz})$, transitional 2 (T2), and transitional 1 (T1) (Supplementary Fig. 2c).

We next analyzed two of the major B-cell subsets subpopulations that have been previously shown to contain the majority of IL-10-producing B cells and were shown to play a role in the regulation of autoimmune diseases ${ }^{20,39}$; T2MzP Bregs (IL-10 ${ }^{+}$ $\left.{ }_{\mathrm{CD}} 24^{+} \mathrm{CD} 21^{\mathrm{hi}} \mathrm{CD} 23^{+}\right)$and $\mathrm{CD} 138^{+}$Bregs $\left(\mathrm{IL}-10^{+} \mathrm{CD} 138^{+}\right)$. The percentages and absolute numbers of both IL-10-positive subpopulations were significantly elevated in slamf $5^{-1-}$ mice compared to WT on day 15, both in the spleen (Fig. 2e, f) and in the LNs (Fig. 2g, h). At the start of the recovery phase, on day 26, an increase in the IL-10-producing Breg populations was detected in the spleen (Supplementary Fig. 2d, e), but not in the LNs of slamf $5^{-1-}$ mice (Supplementary Fig. 2f, g). The elevation in Breg numbers was specific to the disease process since no change in Breg populations was observed in the spleens of WT and slamf5 $5^{-1-}$ that were treated with adjuvant (CFA) alone (Supplementary Fig. 2h, i).

T2MzP B cells, which contain a subpopulation with the potential to become IL-10-producing cells, are mostly located in the spleen; however, T2MzP Bregs $\left(\mathrm{CD} 23^{\text {high }} \mathrm{CD} 21^{\text {high }} \mathrm{IL}-10^{+}\right)$ were detected in the LNs of EAE mice (Supplementary Fig. 3a) and were previously reported in the draining LNs of melanoma tumors ${ }^{40}$. $\mathrm{CD} 138^{+} \mathrm{B}$ cells can be divided into plasmablasts (PB, $\left.\mathrm{CD} 138^{+} \mathrm{CD} 44^{\text {hi }}\right)^{20}$ and plasma cells (PC, CD138 $\left.{ }^{+} \mathrm{CD} 44^{\text {low }}\right)^{41}$. Both subpopulations were shown to consist of IL-10-secreting cells and to have an important role in EAE. Therefore, we followed the regulation of these IL-10-expressing subpopulations by SLAMF5 deficiency. As seen in Supplementary Fig. 3b-d, both populations were increased in the spleen and LNs of slamf5 $5^{-1-}$ mice. Therefore, in the rest of the study, we continued analyzing the total IL- $10^{+} \mathrm{CD} 138^{+}$population.

SLAMF5 deficiency can increase Breg numbers through either direct intrinsic signaling in the Bregs themselves or as a consequence of a change in SLAMF5 signaling in their microenvironment. In order to further unravel the mechanism of SLAMF5, we generated chimeric mice in which SLAMF5 deficiency is mostly restricted to the $\mathrm{B}$ cells, using bone marrow (BM) of JHT mice. These mice lack the $\mathrm{J}_{\mathrm{H}}$ gene, leading to disruption in B-cell development, and resulting in a lack of early and mature B-cell populations ${ }^{42}$. Recipient WT-CD45.1 mice were irradiated and transplanted with a BM mixture of $80 \%$ JHT BM: $20 \%$ slamf5 $^{-1-}$ or WT BM (Supplementary Fig. 4a). Chimerism and restriction of SLAMF5 deficiency mostly to the B-cell populations was confirmed (Supplementary Fig. $4 \mathrm{~b}-\mathrm{d}$ ).

EAE was then induced in the JHT-chimeric mice and the disease course followed for 15 days. The clinical manifestation in the mice reconstituted with $20 \%$ WT cells, typically appeared 10 days after disease induction, increasing in severity and reaching a mean clinical score of $3.3 \pm 0.07$ by day 15 , with disease incidence of $100 \%$. The mice reconstituted with $20 \%$ slamf $5^{-1-}$ cells manifested the disease on day 10 , as well, but with a milder motor dysfunction with a reduced maximal mean score of $2.7 \pm 0.21$ by day 15 , with $95 \%$ of the mice exhibiting the disease (Fig. 2i and Supplementary Fig. 4e). A significant overall difference was evident in the AUC (inset in Fig. 2i). The disease induction in the JHT:WT chimeras was similar to the development of the disease in wild-type mice (Fig. 1a) and to the disease observed when WT BM cells were transplanted into a WT recipient (Supplementary Fig. 4f).

Similar to the fully slamf $5^{-1-}$ mice, we found a significant increase of T2MzP and CD138 ${ }^{+}$Bregs in the spleen (Fig. 2j, k). The LNs of the chimeric mice were small to undetectable, and therefore the LNs of four to five mice with matching scores were combined for analysis. An elevation was seen in T2MzP and $\mathrm{CD} 138^{+}$Bregs percentages and numbers in these chimeric mice (Fig. 2l, m). Furthermore, the Breg was significantly elevated in the SC of the $20 \%$ slamf5 $5^{-1-}$ mice (Fig. 2 n) recapitulating the results of the full SLAMF5-deficient mice. These results suggest that SLAMF5 is an intrinsic negative regulator of Breg abundance in the spinal cord and lymphoid tissues during the active phase of the disease.

SLAMF6 does not play a role in Breg accumulation during EAE. Some of the roles of SLAMF5 overlap with those of other members of the SLAM family. SLAMF6 (Ly108 in mice, NTB-A in humans) regulates naive B-cells maintenance ${ }^{43}$ and the production of autoantibodies ${ }^{44}$. We, therefore, wished to determine whether regulation of Breg accumulation is mediated exclusively by SLAMF5 or whether an additional member of the family, SLAMF6 may have a similar function. SLAMF6 deficiency partially protected mice from EAE, but these mice showed a higher disease incidence of 95\% (Supplementary Fig. 5a-c) compared to the slamf5 $5^{-1-}$. However, lower levels of Bregs were detected in the SC of slamf6 ${ }^{-1-}$ compared to the WT (Supplementary Fig. 5d), and no differences in the levels and numbers of total Bregs in the spleen (Supplementary Fig. 5e, f) and LNs (Supplementary Fig. 5g, h) were observed. Effector T-cell markers were lower in the LNs of slamf6-I- mice compared to WT but were 
a Spinal Cord - Bregs - Day 15

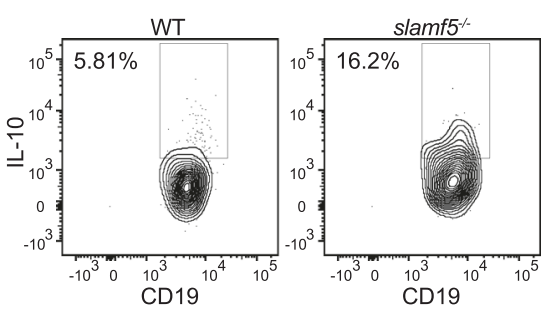

b

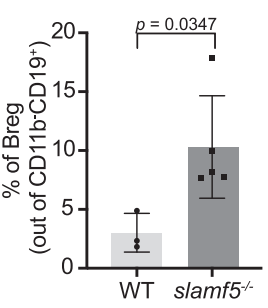

c Periphery - Bregs - Day 15

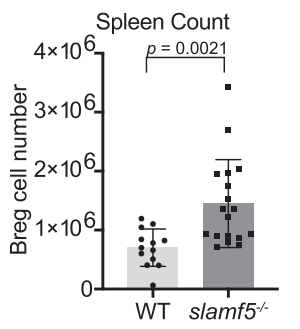

d

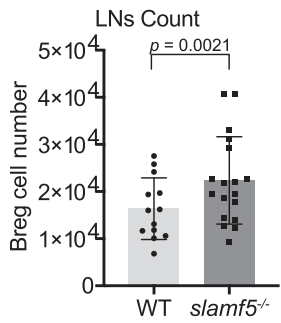

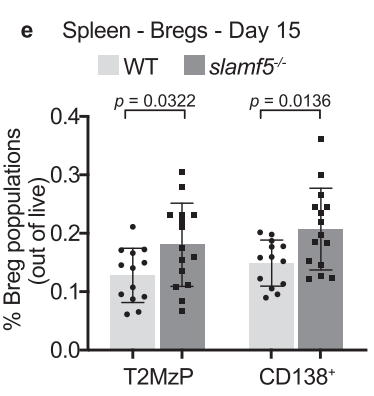

$\mathbf{f}$

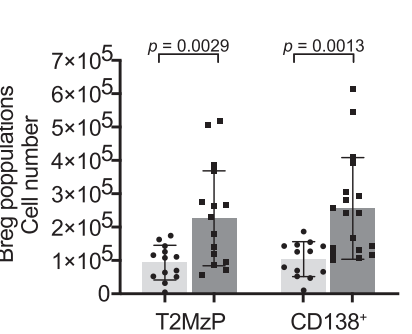

g LNs - Bregs - Day 15 WT slamf5 ${ }^{-1-}$
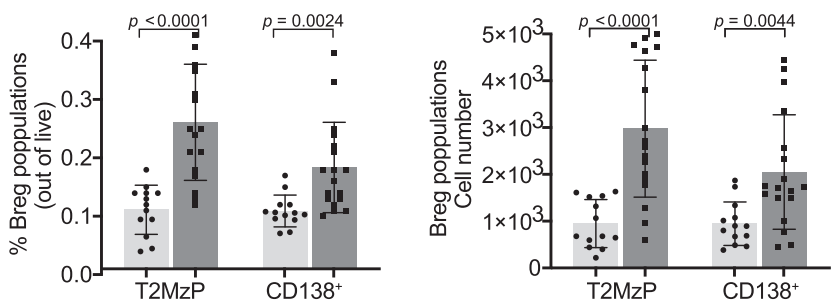
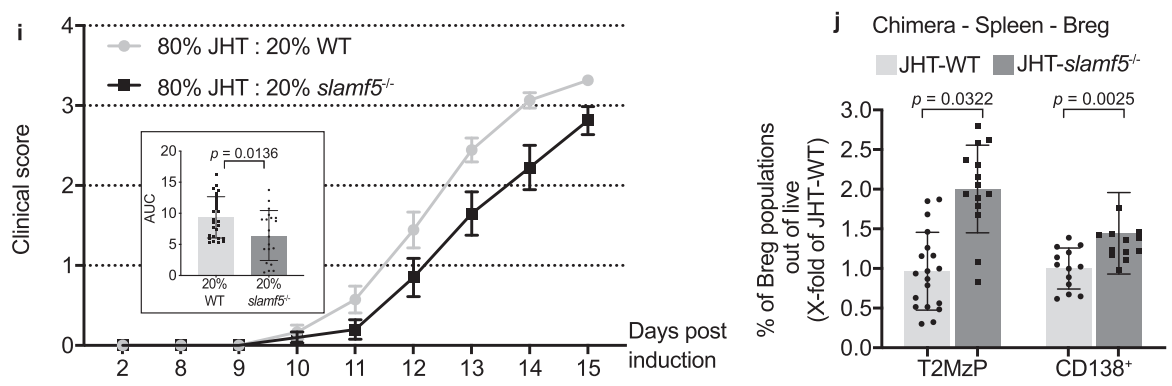

$\mathbf{k}$

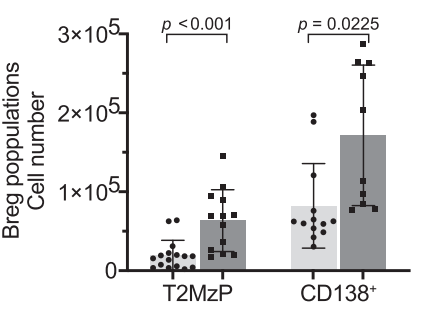

n Chimera - Spinal Cord- Breg

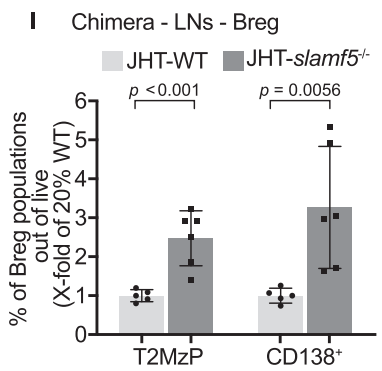

m

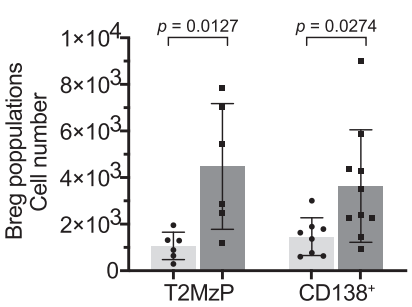

Fig. 2 SLAMF5 deficiency regulates Breg levels. EAE (MOG $\mathbf{3 5}_{\mathbf{3 5}}$ ) was induced in WT and slamf5-/- mice. a, b On day 15 , the spinal cords were analyzed the for Breg population according to the following markers: CD45+CD11b-CD19+CDIL-10+; $\mathbf{a}$ representative dot plot, and $\mathbf{b}$ bar graph showing the mean

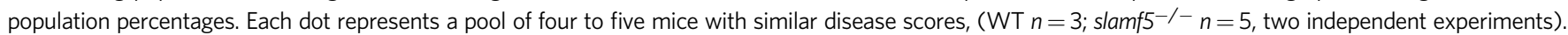
c-h On day 15 , spleens and lymph nodes (LNs) were analyzed. Total Breg (CD19+IL-10 ${ }^{+}$) cell numbers in (c) the spleen and (d) LNs (WT $n=13$; slamf5 ${ }^{-/-} n=$ 18, two independent experiments), subpopulations of T2-MzP Breg (CD19+IL-10+CD24+CD21+CD23+) and CD138+ Bregs $($ CD19+IL-10+CD138+) were analyzed for percentages and cell number in (e, f) the spleen (WT $n=13$; slamf5 ${ }^{-/} n=15$, two independent experiments) and (g, $\left.\mathbf{h}\right)$ LNs (WT $n=13$; slamf5 $-/-$ $n=18$, two independent experiments) (i-n) CD45.1 WT mice were lethally irradiated and injected with BM consisting of $80 \%$ JHT BM and $20 \%$ WT-CD45.2 or slamf5-/- BM. Following BM reconstitution, EAE was induced, and mice were followed for 15 days. i Mean clinical scoring of the disease. Inset depicts the area under the curve for days $0-15$ (20\% WT $n=24 ; 20 \%$ slamf5 $5^{-/} n=20$, three independent experiments). On day 15 , Breg populations were analyzed in the

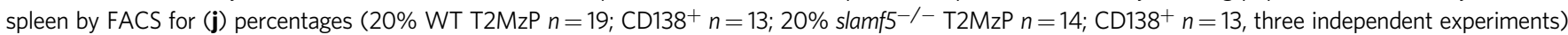

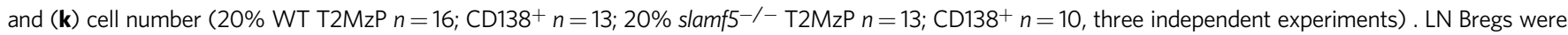
analyzed for their (I) percentage (each dot represents four to five mice with a similar score, $20 \% \mathrm{WT} n=6 ; 20 \%$ slamf5 ${ }^{-/}-n=5$, three independent

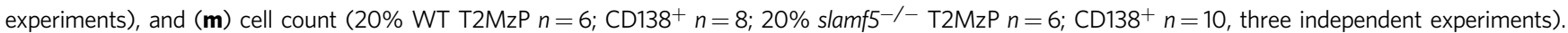
n Spinal cord Bregs (each dot represents four to five mice with a similar score, $20 \%$ WT $n=3 ; 20 \%$ slamf5-1- $n=4$, two independent experiments) were analyzed. Data expressed as mean \pm s.d. (b-h, i inset, $\mathbf{n}$ ) and mean \pm s.e.m. (i). Unpaired Student's $t$ test with $95 \%$ confidence levels two-tailed (b-h, j-m) or onetailed (n), Mann-Whitney test (i insets). Gating strategy for $\mathbf{a}, \mathbf{b}, \mathbf{n}$ is shown in Supplementary Fig. 2a, gating strategy for a-d, e-h, j, $\mathbf{k}$ is shown in Supplementary Fig. $2 b$. 
similar in the spleen (Supplementary Fig. 5i, j), suggesting changes in T-cell function in these mice. Further examination of the role of SLAMF6 in B cells during EAE was performed using JHT-slamf6 ${ }^{-1-}$ chimeric mice, where SLAMF6 deficiency was mostly restricted to B cells. Disease progression showed no difference between JHT-WT and JHT-slamf6-l- mice (Supplementary Fig. 4k). Similar to the full slamf6 ${ }^{-1-}$ mice, no change in Breg levels and numbers was seen in the JHT-slamf6-l- mice compared to JHT-WT (Supplementary Fig. 5l, m). These results suggest that SLAMF6 does not regulate the Breg populations in EAE.

SLAMF5 is highly expressed on Bregs. To follow the role of SLAMF5 in Bregs, we analyzed SLAMF5 expression on different splenic B-cell populations derived from EAE-induced mice. The different subpopulations of B cells were evaluated for SLAMF5 expression on either IL-10-positive (Bregs) or IL-10-negative (non-Breg) populations. Increased levels of SLAMF5 were detected on Bregs compared to its expression on their non-Breg $\mathrm{B}$-cells counterparts, with the highest levels on the T2MzP Bregs (Fig. 3a, b). High levels of SLAMF5 were also detected on Bregs derived from WT mice injected only with CFA relative to nonBreg populations (Supplementary Fig. 6a).

IL-10 staining requires in vitro activation, in which cells are stimulated with the TLR4 agonist lipopolysaccharide (LPS). To verify that the high SLAMF5 expression levels on Bregs did not result from the in vitro stimulation, SLAMF5 levels were analyzed on Vert-x mice which express a GFP reporter on the IL-10 gene, which eliminates the need for TLR4 activation ${ }^{45}$. T2MzP Bregs expressed higher levels of SLAMF5 compared to their IL-10 neg counterparts in both EAE-induced and healthy Vert-x mice (Fig. 3c, d). In addition, although Breg frequencies were higher in mice induced for EAE (Fig. 3e), Bregs from EAE-induced or healthy Vert-x mice expressed similar levels of SLAMF5. This higher SLAM5 expression was also observed on total Bregs compared to total non-Bregs (Supplementary Fig. 6b, d) and CD138 Bregs compared to non-Breg CD138 cells (Supplementary Fig. 6c, d), suggesting that Bregs highly express SLAMF5 regardless of disease or TLR activation.

To further explore the effect of TLR activation on SLAMF5 expression by Breg, its expression was analyzed on in vitro generated Bregs. B cells from naïve mice were incubated for 24 or $72 \mathrm{~h}$ in the presence of LPS. An increase in the Breg population was observed throughout the experiment, while IL-10 neg B-cell levels were reduced (Supplementary Fig. 6e). These Bregs expressed higher levels of SLAMF5 compared to non-Bregs cultured under the same conditions (Supplementary Fig. 6f). However, SLAMF5 expression levels did not change during the incubation time (Supplementary Fig. 6f) on either population, indicating that LPS does not affect SLAMF5 expression levels, further suggesting that SLAMF5 is an intrinsic feature of regulatory B cells.

SLAMF5 negatively regulates Breg function. Next, we wished to determine whether SLAMF5 regulates Breg functionality. Analysis of IL-10 expression in WT and slamf5 ${ }^{-1-}$ Bregs revealed similar levels of this cytokine in both populations (Supplementary Fig. $6 \mathrm{~g}, \mathrm{~h}$ ). To investigate Breg functionality, a Breg suppression assay was performed. WT or slamf5 $5^{-1-}$ transitional 2 (T2) B cells, containing the T2MzP Breg population, were cultured with WT $\mathrm{CD} 25^{-} \mathrm{CD} 4^{+} \mathrm{T}$ cells at $1: 1$ ratio. As previously shown ${ }^{46}$, the suppression of effector T-cell function is partially mediated by IL10 secretion (Supplementary Fig. 7a). The abundance of Bregs in the slamf5 $5^{-1-}$ group was twofold higher than Bregs in the WT T2 $\mathrm{B}$ cells. Therefore, to normalize the number of Bregs in the two groups, WT or slamf5 $5^{-1-}$ T2 cells were cultured with WT CD25$\mathrm{CD}^{+} \mathrm{T}$ cells at a 2:1 ratio. After $72 \mathrm{~h}$, cells were analyzed for the expression of the cytokines IL-17 and IFN $\gamma$. T cells cultured with slamf $5^{-1-}$ B cells showed a significantly lower level of the cytokines IL-17 (Fig. 3f, g and Supplementary Fig. 7b) and IFN $\gamma$ (Fig. 3h, i and Supplementary Fig. 7c), suggesting stronger suppression by slamf5-1- T2 B cells of T-cell differentiation relative to wild-type.

The reduction of $\mathrm{T}$-cell cytokine expression in the presence of slamf5 $5^{-1-} \mathrm{T} 2 \mathrm{~B}$ cells may result from insufficient T- and B-cell interactions through SLAMF5 rather than intrinsic changes in Breg functionality. However, while deficiency of SLAMF5 on B cells increased T-cell suppression, SLAMF5 deficiency on the $\mathrm{T}$ cells themselves had no effect on their suppression (Supplementary Fig. 7d, e). In addition, analysis of T-B cell conjugate formation in the suppression assay by analysis of CD19-CD4 doublets $^{43}$, showed similar levels of the doublets when SLAMF5 deficiency was on the B cells or on the T cells (Supplementary Fig. 7f-h). Thus, SLAMF5 deficiency did not impair the interactions between $\mathrm{B}$ cells and T cells and therefore the elevated suppression detected in the presence of SLAMF5-deficient B cells resulted from a factor intrinsic to these $\mathrm{B}$ cells.

Breg survival is regulated by SLAMF5. SLAMF5 was previously shown to regulate the survival of CLL cells, which express high levels of this receptor ${ }^{31}$. Since Bregs express high levels of SLAMF5, we tested whether blocking this receptor interferes with survival signaling in Bregs, as well. To specifically follow the role of SLAMF5 expressed on B cells, we blocked its activity in purified B-cell populations. B cells from EAE-induced WT mice in remission were cultured for $48 \mathrm{~h}$ under IL-10-inducing conditions (LPS) with either isotype control or SLAMF5-blocking antibody ${ }^{30-32}$. Blocking SLAMF5 significantly increased Breg numbers (Fig. 4a) compared to control-IgG. The increase of Breg numbers was not dependent on proliferation (Fig. 4b), but due to an increase of Breg survival (Fig. 4c, d). While the Breg livepopulation was increased, non-Bregs were not affected, suggesting that SLAMF5 specifically suppresses pro-survival signaling in Bregs (Fig. 4e, f).

To further analyze SLAMF5-dependent pathways in Breg populations, we sorted Bregs from the IL-10 GFP Vert-x reporter mice. Sorted Bregs $\left(\mathrm{CD} 19^{+} \mathrm{GFPhi}\right)$ were treated for $24 \mathrm{~h}$ with SLAMF5-blocking or control-IgG antibodies and were analyzed for the mRNA levels of Bcl-2 family members. Increased levels of $\mathrm{Bcl}-2$ and $\mathrm{Bcl}-\mathrm{xl}$ (Fig. $4 \mathrm{~g}$ ) were detected in the SLAMF5-blocked population, suggesting that SLAMF5 restricts Breg numbers via survival signaling pathways.

Blocking of SLAMF5 mitigates EAE and increases Breg levels. We next examined the effect of SLAMF5-blocking in vivo on EAE pathogenesis. WT mice were induced with $\mathrm{MOG}_{35-55}$, and treated with SLAMF5-blocking or IgG-control antibodies, starting on day 7 after disease induction. Injections were repeated on days 9 and 11 , and mice were followed through day 15 (Fig. 4h). Clinical signs of disease appeared in both groups 10-11 days after disease induction, reaching a maximal mean clinical score of $3.45 \pm 0.134$ for the IgG-treated group, at day 15 when disease incidence was $100 \%$. The SLAMF5-blocking-treated group reached a maximal mean score of $2.72 \pm 0.195$, and an incidence of $94 \%$. The difference between the two groups was significant, as indicated by the AUC (Fig. 4h, AUC in the inset, Supplementary Fig. 8b). This was accompanied by significantly reduced weight loss in mice treated with SLAMF5-blocking antibody (Fig. 4i, AUC in inset). Analysis of splenic Breg populations at the end of the experiment showed that blocking SLAMF5 in EAE-induced (Fig. 4j) or 


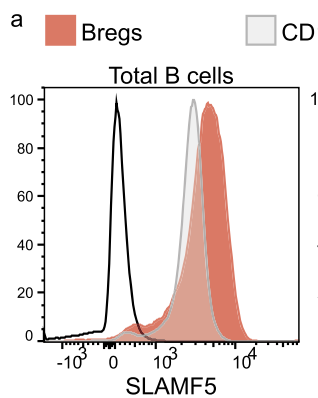

CD19+IL-10 ${ }^{\text {neg }} \square$ slamf5 $^{--}$
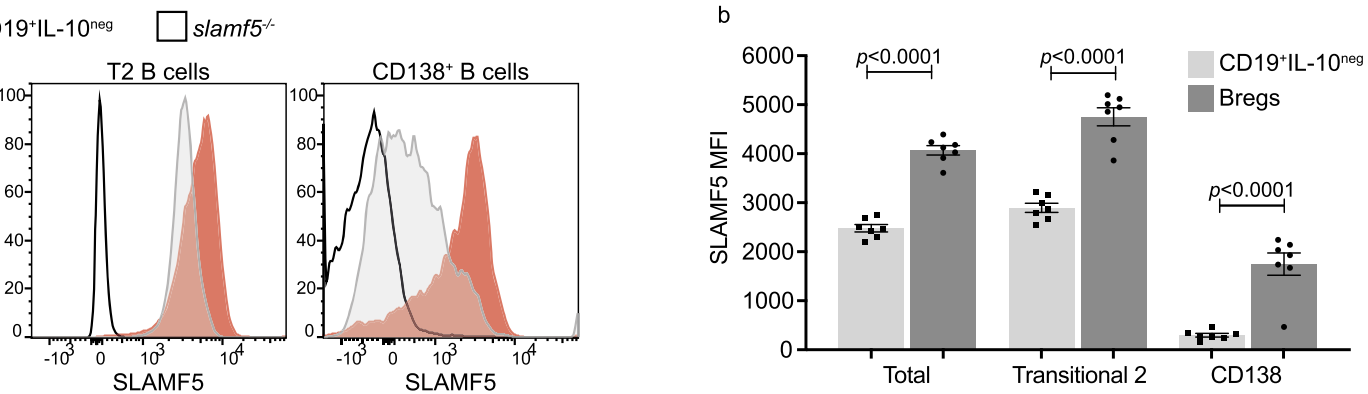

T2MzP B cells - IL-10 GFP Reporter mice
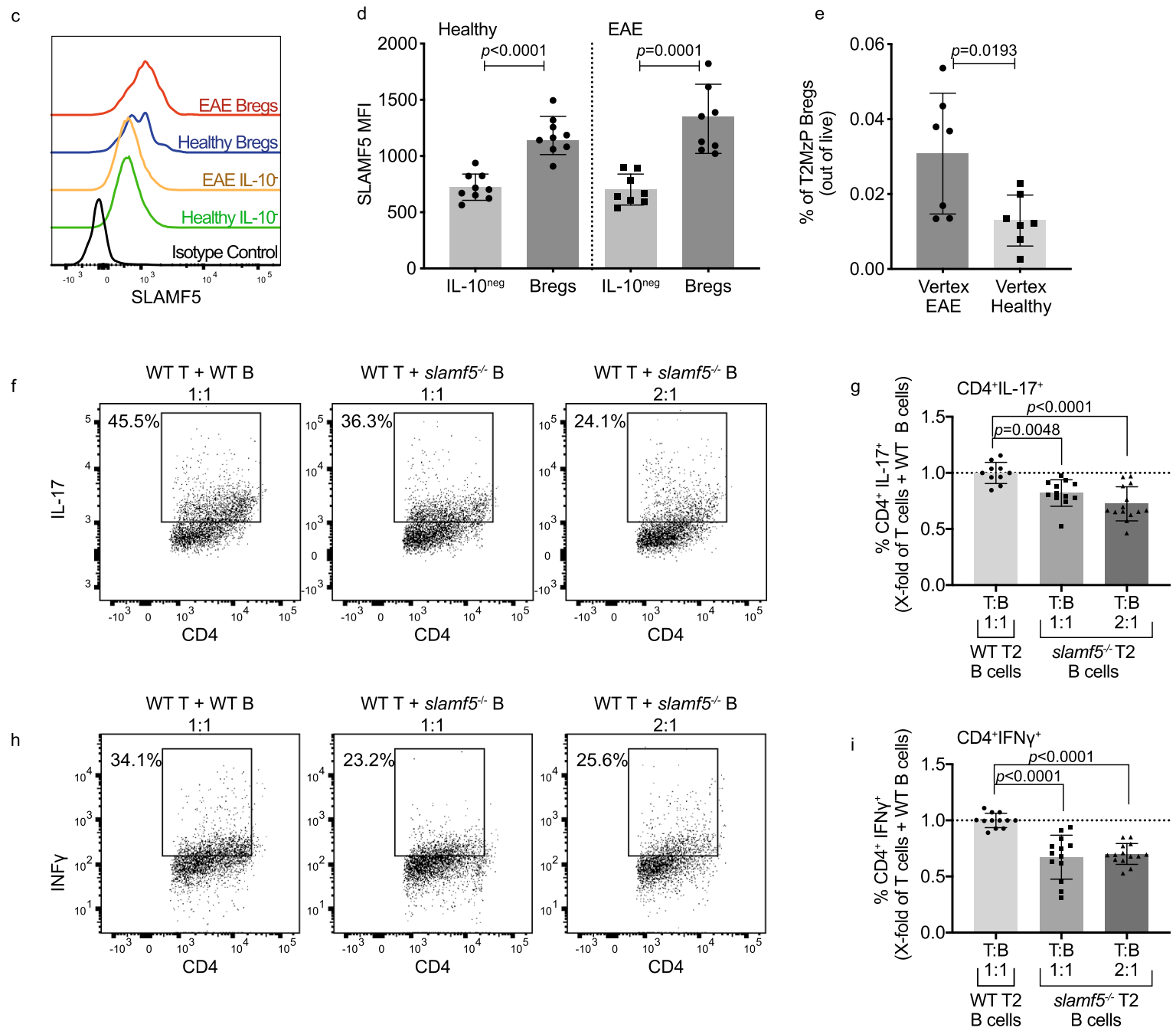

Fig. 3 SLAMF5 is highly expressed on Bregs and regulates their functionality. $\mathbf{a}$, $\mathbf{b}$ Analysis of SLAMF5 expression on splenic regulatory $B$ compared to non-Bregs (B cells IL-10neg) of WT EAE (MOG $35-55$ )-induced mice. a Representative histograms, and $\mathbf{b}$ bar charts showing the SLAMF5 MFI on B-cell subpopulations according to the following markers: total B cells $\left(\mathrm{CD} 19^{+}\right)$, T2 B cells $\left(\mathrm{CD} 19^{+} \mathrm{CD} 24^{+} \mathrm{CD} 21^{+} \mathrm{CD} 23^{+}\right)$, and $\mathrm{CD} 138^{+} \mathrm{B}$ cells $\left(\mathrm{CD} 19^{+} \mathrm{CD} 138^{+}\right)$. Populations are divided into IL-10+ (Bregs) and IL-10neg (non-Bregs) (non-Bregs $n=7$; Bregs $n=7$, two independent experiments). c-e Spleens of Vert-x mice, healthy or EAE-induced, were harvested and analyzed for SLAMF5 expression on IL-10+ or IL-10neg T2MzP cells. c Representative histogram and d bar graph showing SLAMF5 MFI. (EAE: Bregs $n=8$; non-Bregs $n=8$; healthy: Bregs $n=9$ non-Bregs $n=9$, two independent experiments). e Spleens were analyzed for percentages of T2MzP Bregs ( $\mathrm{IL}-10^{+}$) (healthy $n=7$; EAE $n=7$, two independent experiments). $\mathbf{f}-\mathbf{i}$ Sorted $\mathrm{CD} 4+{ }^{+} \mathrm{CD} 25^{-}$splenic $\mathrm{T}$ cells of WT EAE-induced mice were co-cultured 1:1 or 2:1 for $72 \mathrm{~h}$ in the presence of anti-CD3 and anti-CD28 Abs with WT or slamf5-/- sorted splenic transitional 2 B cells (CD19+CD23+CD21+). Cells were analyzed for $(\mathbf{f}-\mathbf{g})$ IL-17 expression, shown as (f) representative dot plots, and (e) bar graph (WT T:WT B 1:1 $n$ $=10 ;$ WT T:slamf5 ${ }^{-/}$- B 1:1 $n=12 ;$ WT T:slamf5 ${ }^{-/-}$B 2:1 $n=14$, five independent experiments) and (h, i) IFN $\gamma$ expression, shown as (h) representative dot plots, and (i) bar graph. (WT T:WT B 1:1 n =12; WT T:slamf5-/- B 1:1 $n=13$; WT T:slamf5 $-/-$ B 2:1 $n=14$, five independent experiments). Data expressed as mean \pm s.e.m. (b) and mean \pm s.d. (d, e, g, i). Unpaired Student's $t$ test with $95 \%$ confidence levels two-tailed (b, d, e) or ordinary one-way ANOVA with Dunnett multiple comparison tests $(\mathbf{g}, \mathbf{i})$. Gating strategy for $\mathbf{a}$ and $\mathbf{b}$ is shown in Supplementary Fig. $2 b$, gating strategy for $\mathbf{c}$ and $\mathbf{d}$ is shown in Supplementary Fig. 6d, gating strategy for $\mathbf{h}$ and $\mathbf{i}$ is shown in Supplementary Fig. 1b (I., III.). 

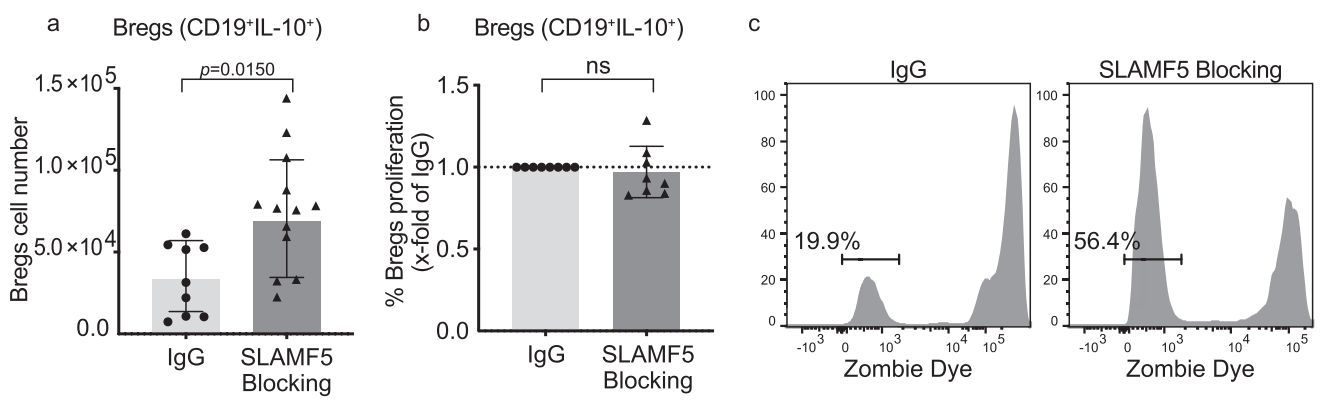

d $\quad$ Bregs $\left(C D 19^{+} \mid L-10^{+}\right)$
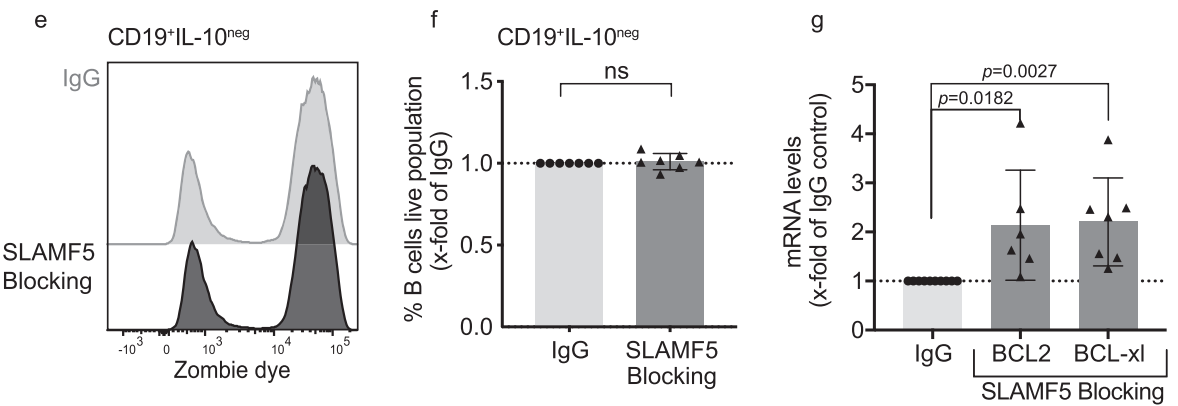

h

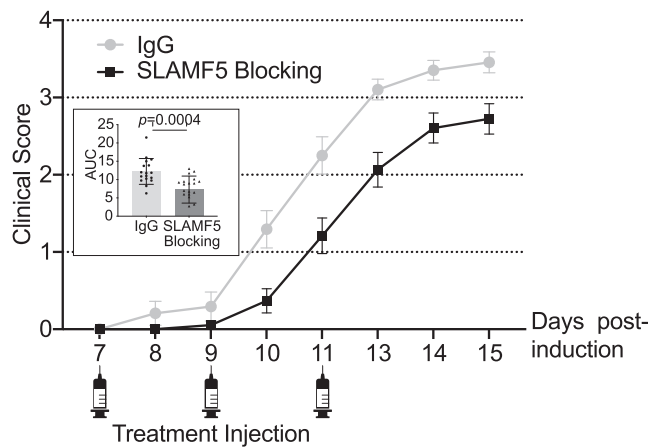

i
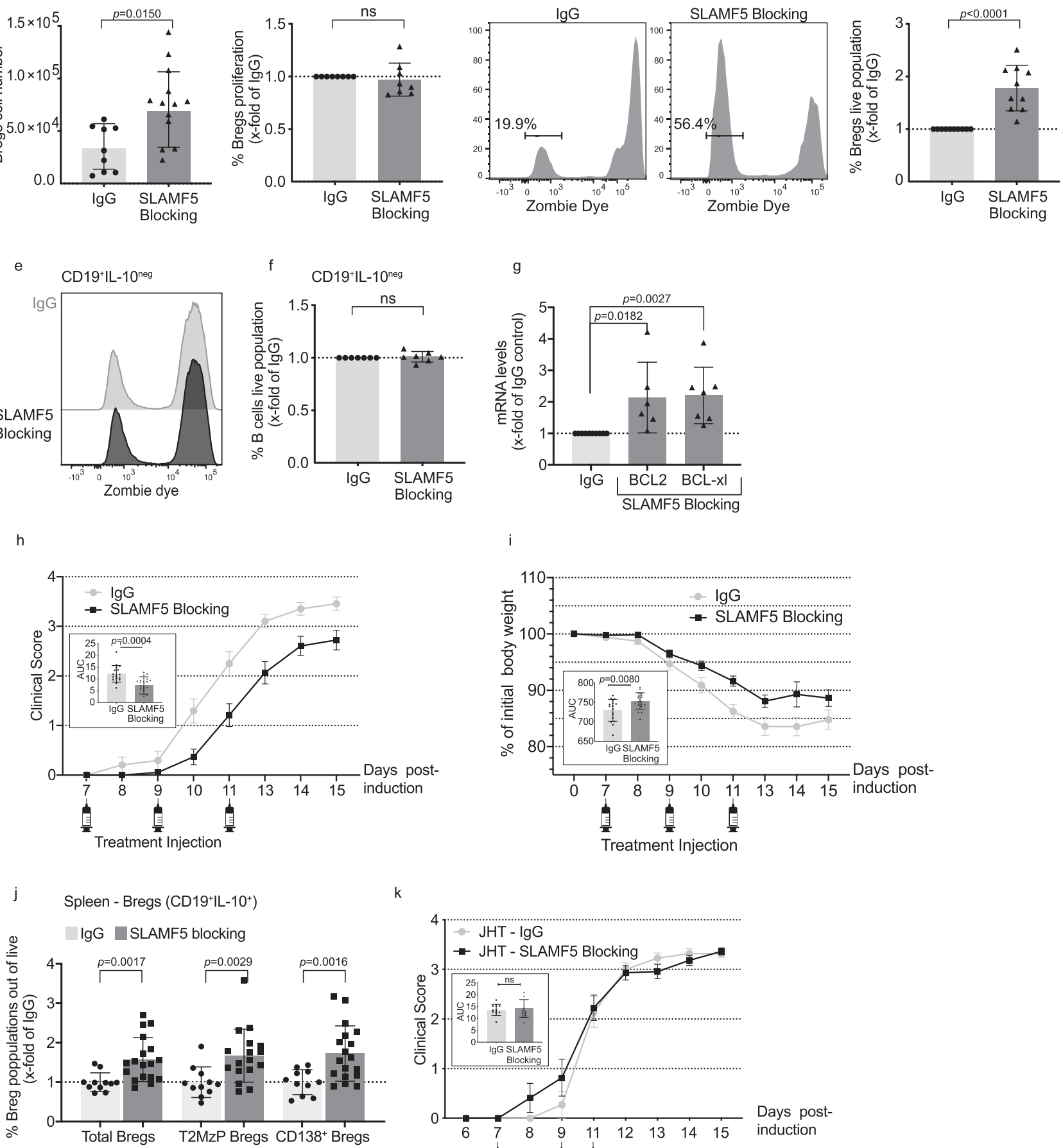

k

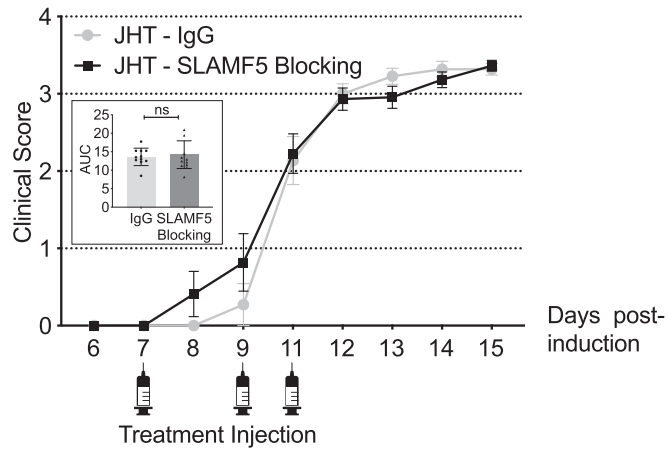

healthy mice (Supplementary Fig. 8c) upregulated the levels of total Bregs and the Breg subpopulations $\mathrm{T} 2 \mathrm{MzP}$ and $\mathrm{CD} 138^{+}$ (Fig. 4j), suggesting that SLAMF5-blocking downregulates Breg numbers in health and disease.

Since SLAMF5 is expressed on several immune cells, its blockade can affect the function of non-B cells. To determine whether the effect of SLAMF5-blocking may also stem from an effect on the non-B-cell populations, EAE-induced JHT mice were treated with SLAMF5-blocking or IgG antibodies. Clinical signs of disease appeared in both treatment groups 7-8 days after disease induction, and both groups reached a similar maximal mean clinical score of $3.27 \pm 0.104$ for the IgG-treated group and
$3.36 \pm 0.07$ for the anti-SLAMF5-treated group. In both groups, a disease incidence of $100 \%$ was reached. Overall, no significant differences were observed over the disease course (Fig. $4 \mathrm{k}$ and AUC in the inset, Supplementary Fig. 8d). These results suggest that blocking of SLAMF5 leads to an improved clinical score and outcome for EAE mice by boosting their regulatory B-cell levels and that the beneficial effect of SLAMF5-blocking is mediated mainly through its effect on B cells.

Blocking SLAMF5 increases c-Maf. To attain a deeper understanding of the role of SLAMF5 in Bregs, total cellular mRNA 
Fig. 4 SLAMF5 blocking increases Breg survival in vitro and increases Breg numbers in vivo. a-f Splenic $B$ cells derived from EAE-induced WT mice were cultured and treated with LPS and either SLAMF5-blocking or lgG-control antibodies for $48 \mathrm{~h}$, with PMA, ionomycin, and monensin for the final $5 \mathrm{~h}$. Cells were collected, and the survival of Bregs and non-Breg B cells (CD19-IL-10neg) was analyzed using Zombie dye by flow cytometry; a Breg cell numbers ( $\lg n=9$; SLAMF5-blocking $n=15$, five independent experiments), b Breg proliferation (IgG $n=8$; SLAMF5-blocking $n=8$, three independent experiments), c, d Breg survival showing (c) representative histograms of Zombie dye staining (live dead staining), and $\mathbf{d}$ percentage of live (Zombie ${ }^{\text {neg })}$ Breg shown as $x$-fold of $\operatorname{lgG}$ ( $\operatorname{lgG} n=10$; SLAMF5-blocking $n=10$, four independent experiments). e, $\mathbf{f}$ Non-Breg B-cell survival: e representative histograms of Zombie dye staining and (f) graph summarizing the percentage of live (Zombieneg) B cells (IgG $n=7$; SLAMF5-blocking $n=7$, three independent experiments). $\mathbf{g}$ B-cell pool of two to three EAE-induced Vert-x mice was sorted for Bregs (CD19+GFP ${ }^{+}$), cultured, and treated with SLAMF5blocking $\mathrm{Ab}$ or $\mathrm{lgG}$-control for $24 \mathrm{~h}$. Cells were analyzed for mRNA levels of Bcl-2 and Bcl-xl by qPCR analysis. Data are shown as $x$-fold of treatment compared to IgG-control. Each dot represents a combination of two to three score-matched mice (Bcl-2: IgG $n=6$; SLAMF5-blocking $n=6$, two independent experiments, Bcl-xl: IgG $n=7$; SLAMF5-blocking $n=7$, two independent experiments). h-j EAE was induced in WT mice. On days 7, 9 , and 11 the mice were injected i.v. with $30 \mu \mathrm{g}$ SLAMF5-blocking Ab or lgG. h Daily mean clinical scoring of the disease (SLAMF5-blocking $n=18$; IgG $n=17$; three independent experiments). i Mean weight loss during the disease course shown as percent of initial body weight. Inset depicts the area under the curve for days 0-15. (SLAMF5-blocking $n=19$; IgG $n=17$; three independent experiments). j On day 15, spleens were collected and analyzed for Breg populations; total Bregs $\left(\mathrm{CD} 19^{+} \mathrm{IL}-10^{+}\right)$, T2MzP $\left(\mathrm{CD} 19^{+} \mathrm{IL}-10^{+} \mathrm{CD} 24^{+} \mathrm{CD} 23^{+} \mathrm{CD} 21^{+}\right)$and $\mathrm{CD} 138^{+}$Bregs $\left(\mathrm{CD} 19^{+} \mathrm{IL}-10^{+} \mathrm{CD} 138^{+}\right)$. (SLAMF5-blocking: total $n=18$ $\mathrm{T} 2 \mathrm{MzP} n=17 ; \mathrm{CD} 138 n=18$; IgG: total $n=11 ; \mathrm{T} 2 \mathrm{MzP} n=11 ; \mathrm{CD} 138 n=11$, three independent experiments). $\mathbf{k}$ EAE was induced in JHT mice. On days 7, 9, and 11 the mice were injected i.v. with $30 \mu \mathrm{g}$ SLAMF5-blocking Ab or IgG. Daily mean clinical scoring of the disease. Insets depict the area under the curve for days 0-15. (SLAMF5-blocking $n=11 ; \lg n=11$; two independent experiments. Data expressed as mean \pm s.d. (a-d, $\mathbf{f}, \mathbf{g}, \mathbf{h}, \mathbf{i}$ insets, $\mathbf{j}, \mathbf{k}$ inset) and mean \pm s.e.m. (h, i, k). Unpaired Student's $t$ test with $95 \%$ confidence levels two-tailed (a, j). Ratio paired Student's $t$ test with $95 \%$ confidence levels two-tailed $(\mathbf{b}, \mathbf{d}, \mathbf{f}, \mathbf{g})$, Mann-Whitney test (h, $\mathbf{i}, \mathbf{k}$ insets). The gating strategy for $\mathbf{a}$ is shown in Supplementary Fig. 8a, gating strategy for $\mathbf{j}$ is shown in Supplementary Fig. 2b.

levels in SLAMF5-blocked Bregs were analyzed by RNA-Seq. Sorted Bregs of EAE-induced Vert- $x$ mice (Supplementary Fig. 8e) were incubated with the SLAMF5-blocking or IgG antibodies. After $24 \mathrm{~h}, \mathrm{mRNA}$ was extracted, prepared for sequencing, and analyzed. Significant changes (absolute change $>2 ; P$ value $<$ 0.05 ) in 277 genes were found, with 141 genes downregulated and 136 genes upregulated compared to the IgG-control group. The differential regulation is summarized in Fig. 5a. Analysis using the EnrichR analysis software identified various pathways that were enriched in the treatment group (Fig. 5a, b). Blocking SLAMF5 resulted in changes associated with cell survival, e.g., apoptosis; NF- $\kappa \mathrm{B}$ pathways, IL-10 signaling pathways, e.g., T-cell activation; IL-10 anti-inflammatory pathways, and others (Fig. 5a, b).

We chose to focus on the maf gene, encoding the transcription factor (TF) c-Maf. This TF is essential for IL-10 production in $\mathrm{T}$ cells and macrophages ${ }^{47}$ and was recently shown to be crucial for IL-10 production in regulatory $\mathrm{B}$ cells ${ }^{48}$. Maf expression was dramatically increased in one of the RNA-seq samples (Fig. 5a). This phenotype was further validated using $\mathrm{qPCR}$, confirming that blocking of SLAMF5 in vitro increases the expression of this essential TF (Fig. 5c). We further analyzed the c-Maf co-TF $\mathrm{AhR}^{49}$, which was recently shown to be essential for IL-10 production in Bregs ${ }^{50}$, and found that its mRNA levels were elevated under SLAMF5-blocking conditions (Fig. 5d).

We next followed the in vivo regulation of c-Maf expression by SLAMF5. EAE-induced mice were treated with SLAMF5-blocking or IgG-control antibodies on days 7, 9, and 11 following disease induction. Consistent with the in vitro data, increased levels of cMaf protein were detected in the regulatory $\mathrm{B}$ cells of the mice treated with SLAMF5-blocking antibody (Fig. 5e, f). This increase was specific to the EAE-derived Bregs and was not observed in the IL- $10^{\text {neg }}$ B-cell population (Fig. 5f). Furthermore, Bregs and non-Bregs derived from naive mice treated with SLAMF5blocking antibody did not show any change in their c-Maf levels (Supplementary Fig. 8e), indicating that the effect of SLAMF5blocking on c-Maf is specific to cells derived from the diseased mice, and suggesting that SLAMF5 negatively regulates c-Maf and AhR expression during EAE.

SLAMF5 regulates human Breg survival and c-Maf expression. We next wished to determine whether SLAMF5 plays a regulatory role in human Bregs. Analysis of SLAMF5 expression on Bregs derived from the peripheral blood (PB) of healthy donors revealed that human Bregs express high levels of this receptor compared to IL-10-negative B cells (Fig. 6a, b). To determine whether SLAMF5 plays a role in human Breg survival, human PB B cells from healthy donors were treated with anti-SLAMF5-blocking or IgGcontrol antibodies. Blocking SLAMF5 significantly increased the live-Breg population, while showing no effect on the IL-10-negative B cells (Fig. 6c). Furthermore, an increase in c-Maf was detected in mRNA in total B cells (Fig. 6d) and protein (Fig. 6e, f) levels in Bregs incubated with SLAMF5-blocking antibody.

Multiple sclerosis (MS) patients were shown to have functionally impaired Breg populations ${ }^{51}$. In addition, there is evidence that MS patients exhibit reduced numbers of Bregs ${ }^{52-54}$. Since our results indicate a negative regulatory mechanism on Breg numbers by SLAMF5, we determined whether SLAMF5 expression is altered on Bregs derived from newly diagnosed untreated MS patients. Analysis of SLAMF5 revealed a significant increase of its expression on total Bregs $\left(\mathrm{CD} 19^{+} \mathrm{IL}-10^{+}\right)$, immature Bregs (also termed immature transitional Bregs, $\left.\mathrm{CD} 19^{+} \mathrm{CD} 24^{+} \mathrm{CD} 38^{\mathrm{hi}} \mathrm{IL}-10^{+}\right)^{55}$, and B10 Bregs $\left(\mathrm{CD} 19^{+} \mathrm{CD} 24^{+} \mathrm{CD} 27^{+} \mathrm{IL}-10^{+}\right)^{56}$ compared to healthy donors (Fig. 6g, h). These results suggest that dysregulation of SLAMF5 expression on Bregs may lead to abnormal Breg numbers and function.

\section{Discussion}

Regulatory B cells are important suppressors of inflammation, and their homeostasis must be tightly controlled. Overactivation of regulatory cells may lead to immune deficiencies and susceptibility to pathogens and cancer, while insufficient activation may lead to an uncontrolled immune response and autoimmune diseases ${ }^{57}$. In this study, we identified a fine-tuning mechanism that negatively regulates Breg number and function through the cell surface receptor SLAMF5 in humans and in mice. Our results show that SLAMF5 deficiency significantly mitigates EAE. slamf5 $5^{-/}$mice show a delayed onset of EAE, and significantly milder disease with lower incidence compared to WT mice.

Bregs have a protective role in $\mathrm{EAE}^{58}$, and their numbers and their suppressive functions are increased during the inflammatory phase of autoimmune diseases. During EAE, in the absence of SLAMF5, mice exhibit higher levels of Breg both in the SC and lymphoid organs. Specifically, in the periphery, we show an increase in the accumulation of $\mathrm{T} 2 \mathrm{MzP}$ and $\mathrm{CD} 138+$ Breg 


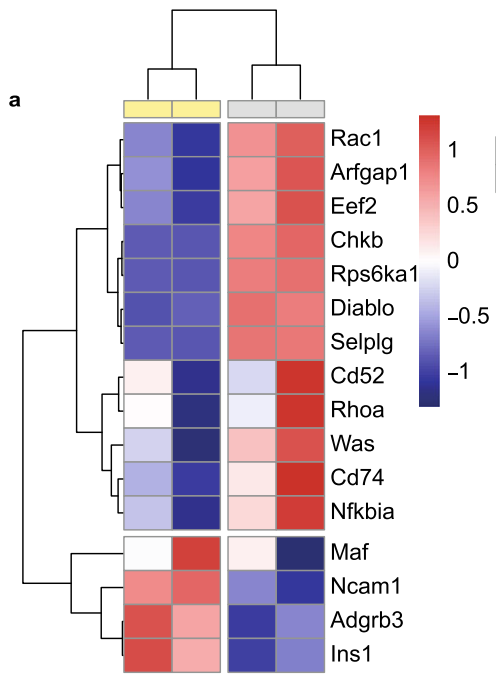

Treatment

SLAMF5 blocking $\lg \mathrm{G} 2 \mathrm{a}$

GATA3 participat

Ion Channels and Their Role in Vascular Endothelum

IL-10 Anti-inflammatory Signaling Pathway

TNF/Stress Related Signaling

Apoptosis signaling pathway

EGF receptor signaling pathway

NFkB activation by Nontypeable Hemophilus influenzae

Toll-Like Receptor Pathway
Biological pathways

Represented regulated genes

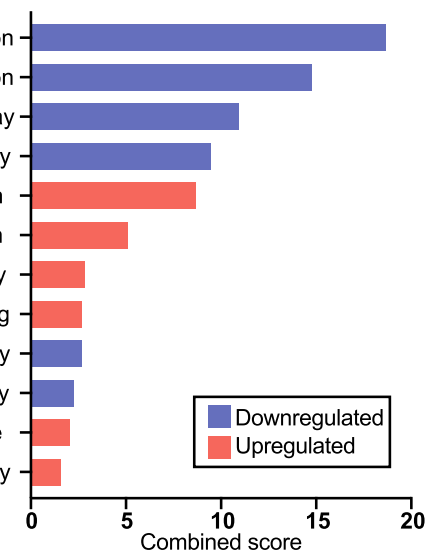

( $Z$ score and $p$-value)
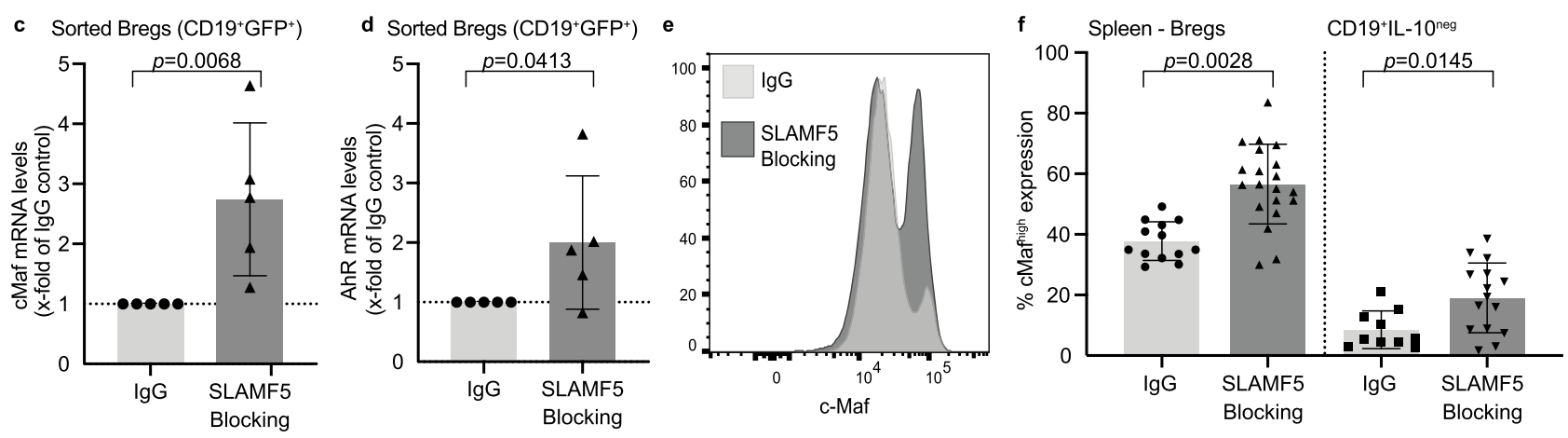

Fig. 5 SLAMF5 regulates the transcription factor c-Maf. B cells derived from EAE-induced Vert-x mice were sorted for Bregs (CD19+GFP + ) and treated for $24 \mathrm{~h}$ with SLAMF5-blocking or lgG-control antibodies. RNA was then extracted from the cells and analyzed. $\mathbf{a}$, $\mathbf{b}$ mRNA-seq analysis of differentially expressed genes in anti-SLAMF5 compared to IgG-treated cells performed in two independent replicates. a Heatmap showing differentially expressed genes, with the $\log 2$ normalized counts standardized for each gene to a mean of zero. The hierarchical clustering of the genes is shown. The expression profile is accompanied by a colored bar indicating the standardized log2 normalized counts. Blue: downregulated; red: upregulated genes. $\mathbf{b}$ Pathway enrichment analysis of 277 genes that were found to be significantly changed (absolute change $>2 ; P$ value $<0.05$ ) was done using the EnrichR platform; the graph shows upregulated (red) and downregulated (blue) pathways ( $\operatorname{gG} n=2$; SLAMF5-blocking $n=2$, each sample is a pool of cells derived of three mice). c qPCR validation analysis of c-Maf mRNA in SLAMF5-blocked Vert-x sorted Bregs (CD19+GFP + ) (IgG $n=5$; SLAMF5-blocking $n=5$. Each dot represents a pool of two to three mice, four independent experiments). d AhR mRNA levels in Vert-x sorted Bregs following SLAMF5-blocking. Data are shown as $x$-fold of the treatment group compared to $\operatorname{lgG}$-control $(\operatorname{lgG} n=5$; SLAMF5-blocking $n=5$. Each dot represents a pool of two to three mice, four independent experiments). e, f EAE was induced in WT mice. On days 7, 9, and 11, the mice were injected i.v. with $30 \mu \mathrm{g}$ SLAMF5-blocking Ab or lgG. On day 15, spleens were collected and analyzed for c-Maf expression by FACS. e Representative histogram showing c-Mafhigh expression of Bregs or nonBregs. $\mathbf{f}$ Bar chart showing c-Maf levels in Bregs and non-Bregs. (non-Bregs: IgG $n=10$; SLAMF5-blocking $n=15$, Bregs: IgG $n=5$; SLAMF5-blocking $n=$ 10, two independent experiments). C-Maf expression was done under the Breg or non-Breg gate as shown in Supplementary Fig. 2c. Data expressed as mean \pm s.d. (c, d, f). Ratio paired Student's $t$ test with $95 \%$ confidence levels two-tailed $(\mathbf{c}, \mathbf{d})$, unpaired Student's $t$ test with $95 \%$ confidence levels twotailed (f). Pathway analysis was done using EnrichR and showing combined score (combined $=\ln (P$ value $) \times z$-score. $P$ value is derived from Fisher's exact test, $z$-score is derived of a modified Fisher exact test.

subpopulations. Treating EAE-induced WT mice with SLAMF5blocking Ab of increased Breg levels and disease mitigation. These findings indicate that SLAMF5 which is highly expressed on Bregs fine-tunes Breg numbers.

SLAM receptors mediate activating-or inhibiting-signals, depending on the cell type and presence of various downstream adaptors. Blocking SLAMF5 leads to an increased expression of anti-apoptotic genes of the BCL-2 family, consequently increasing Breg survival. Our previous results showed that blocking the SLAMF5 receptor expressed on CLL cells leads to a decrease in cell viability, suggesting that the effect of SLAMF5 is cell and disease-specific ${ }^{31}$. In addition, SLAMF5 controls the expression of the maf gene, which encodes the protein c-Maf. c-Maf is a DNAbinding transcription factor that can act as a transcriptional activator or repressor, depending on the binding site and binding partner $^{47}$. c-Maf was recently found to be crucial for IL-10 production in regulatory $\mathrm{B}$ cells ${ }^{48}$. It regulates IL-10 with its co-factor AhR, which was also recently shown to be crucial for IL-10 production in Bregs ${ }^{50}$. We demonstrate that c-Maf and Ahr expression levels are elevated following SLAMF5-blocking. Thus, SLAMF5 controls both the survival of Bregs through the BCL family members and the potential of B cells to differentiate into Bregs by regulating the expression of c-Maf and its co-factor, AhR. We suggest that c-Maf might have a direct role in regulating 


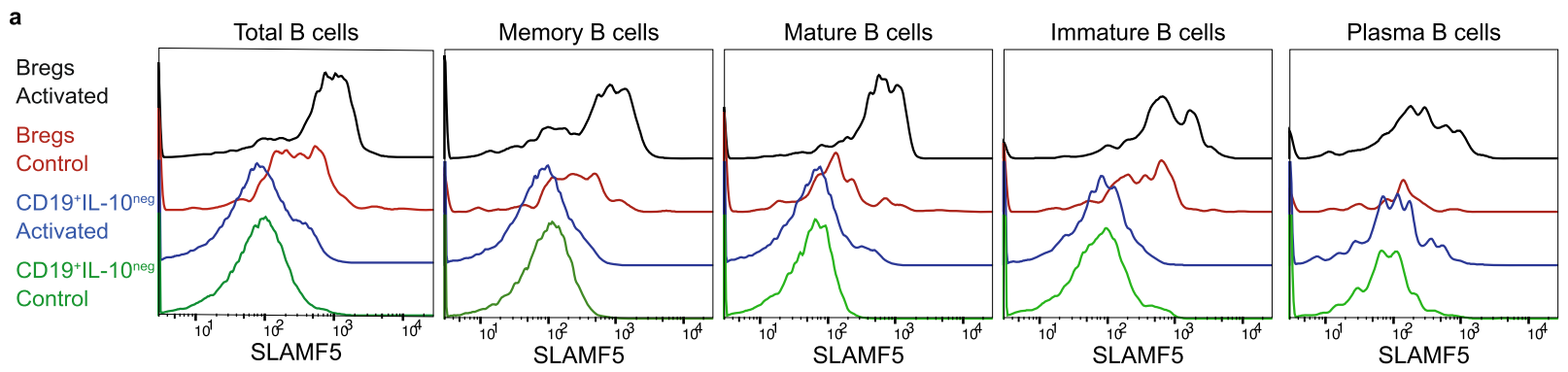

b

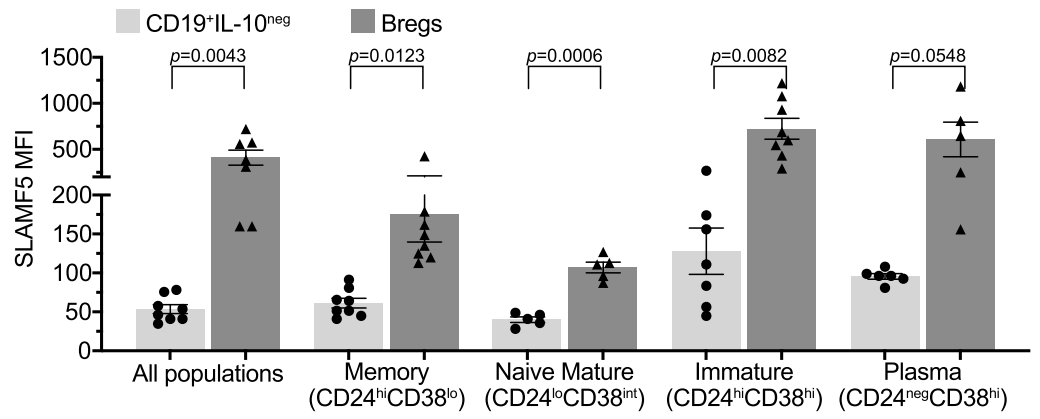

c

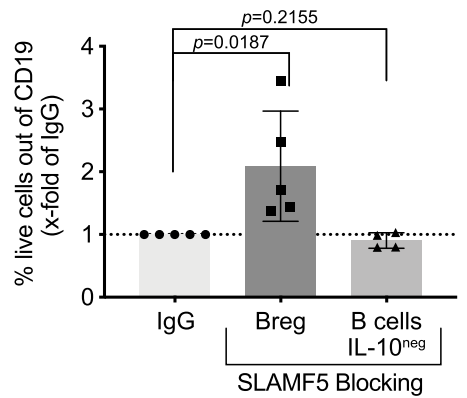

d $\quad \mathrm{CD} 19^{+}$

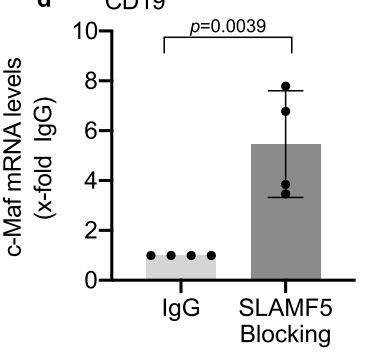

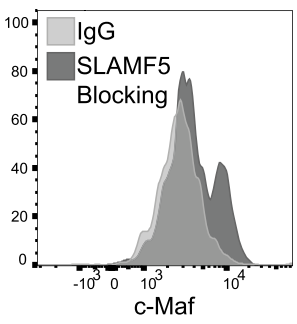

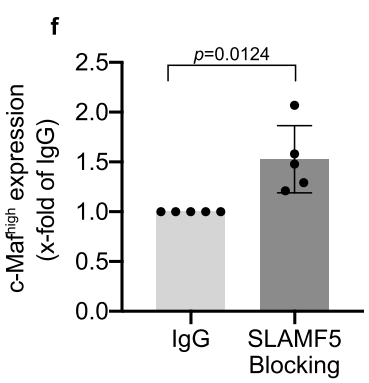

g

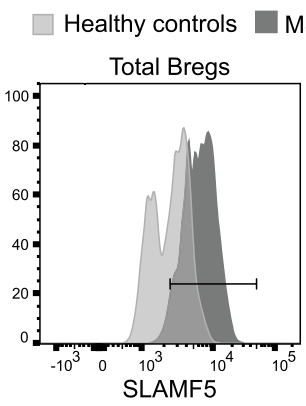

MS new patients

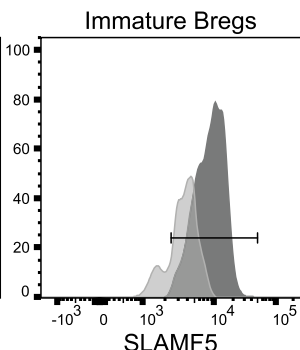

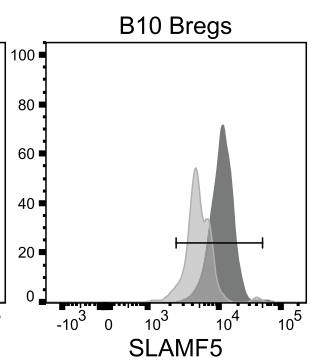

h

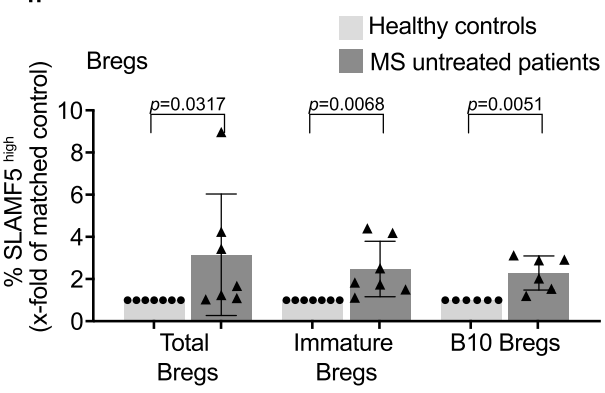

Breg survival by controlling BCL2 expression. Alternatively, its function in these cells might not be related to cell survival, but only to the regulation of IL-10 expression.

While SLAMF5 inhibition showed no effect on the in vitro survival of IL-10-negative B cells, its in vivo effect on these cells cannot be completely excluded. However, since in the $\mathrm{MOG}_{35-55}$ model, B cells do not secrete disease-associated antibodies or proinflammatory cytokines or act as strong APCs, we suggest that the effect of SLAM5 is attributed mainly to the regulatory B cells.

In MS, the B-cell cytokine profile shifts toward a more proinflammatory one, and accordingly, patients exhibit various Breg defects such as irregular numbers $52,53,59$ and impaired function $^{60-62}$. Regulatory B cells of naive, newly diagnosed and untreated, MS patients express significantly higher levels of
SLAMF5 compared to its expression on healthy controls, suggesting a role for SLAMF5 as a negative regulator of Breg levels and activity in the disease. Our results imply that the abnormal increase of SLAMF5 on Bregs derived from untreated MS patients may induce an excessive negative regulation of these cells and may underlie the insufficient Breg response during MS.

In conclusion, we suggest that SLAMF5 can serve as a therapeutic target in MS and other autoimmune diseases. Blocking SLAMF5 can increase the survival of Bregs in these patients, while not affecting the pro-inflammatory B cells. As deficiency of SLAMF5 has almost no effect on the immune repertoire and response in slamf5 $5^{-1-}$ mice ${ }^{29}$, it is likely that SLAMF5 can offer a safe immune-target to boost self-regulatory mechanisms to reduce autoimmunity in patients. 
Fig. 6 SLAMF5 fine-tunes the survival of human Bregs. a, b Healthy human PBMCs were activated for $5 \mathrm{~h}$ with PMA, ionomycin, brefeldin $A$, and monensin or with monensin alone (control) and analyzed by FACS for SLAMF5 expression on B-cell populations: total B cells: CD19+; memory B cells: CD19+ CD24high $C D 38^{\text {low; }}$ naive mature B cells: $C D 19+C D 24^{\text {low }} C D 38$ low; immature B cells: $C D 19+C D 24^{\text {high }} \mathrm{CD} 38^{\text {high; }}$; and plasma B cells: $\mathrm{CD} 19+\mathrm{CD} 24^{\text {low }} \mathrm{CD} 38^{\text {high }}$. Regulatory B-cell subpopulations were analyzed with similar markers under the CD19+IL-10 gate. Gating is shown in Supplementary Fig. 9a. a Representative histograms, and (b) bar charts of SLAMF5 expression on IL-10+ (Breg) and IL-10 neg (non-Breg) populations. (non-Bregs $n=7 ;$ Bregs $n=7 ;$ memory B cells $n$ $=8$; memory Bregs $n=7$; naive B cells $n=5$; naive Bregs $n=5$; transitional B cells $n=7$; transitional Breg $n=8$; plasma B cell $n=6$; plasma Breg $n=5$, four independent experiments). c-f Purified human B cells from healthy donors were treated with SLAMF5-blocking or lgG-control antibody for $48 \mathrm{~h}$. For the last 5 $h$, the cells were activated with PIM and analyzed for (c) cell survival by Zombie dye staining. Bars chart of live Bregs $\left(C D 19^{+} I L-10^{+}\right.$Zombie $\left.{ }^{\text {neg }}\right)$ or non-Breg $B$ cells (CD19+IL-10-Zombie ${ }^{-}$) out of total CD19+. (IgG: Breg $n=5$; non-Breg $=5$, SLAMF5-blocking: Breg $n=5$; non-Breg $n=5$, three independent experiments). $\mathbf{d}$ After $24 \mathrm{~h}$ of treatment, cells were collected for mRNA analysis of c-Maf on total B cells. Results are shown as $x$-fold of treatment compared to IgG-control. (IgG $n=4$; SLAMF5-blocking $n=4$, two independent experiments). e, $\mathbf{f}$ c-Maf expression in Bregs was analyzed by FACS after $48 \mathrm{~h}$. e Representative histogram, and (f) bar chart of c-Maf expression. Results are shown as $x$-fold of treatment compared to lgG-control. (IgG =5; SLAMF5blocking $n=5$, two independent experiments). $\mathbf{g}$, $\mathbf{h}$ Blood samples derived from newly diagnosed untreated multiple sclerosis patients and matched healthy controls were processed, and PBMC were activated for $5 \mathrm{~h}$ with PMA, ionomycin, monensin, and brefeldin A, and stained for SLAMF5 and IL-10 on Breg populations. Total Bregs: CD19+IL-10+; immature Bregs: CD19+IL-10+CD24+CD38+; B10 Bregs: CD19+IL-10 ${ }^{+}, \mathrm{CD}^{+} 4^{+} \mathrm{CD} 27^{+}$. g Representative histograms showing the gate used to define SLAMF5high expression and $\mathbf{h}$ bar charts of SLAMF5high expression on Breg populations presented as fold increase over each sex- and age-matched control. Data expressed as mean \pm s.e.m. (b) or mean \pm s.d. (c, d, f, h). Paired Student's $t$ test with $95 \%$ confidence levels two-tailed (b). Ratio paired Student's $t$ test with 95\% confidence levels two-tailed unpaired Student's $t$ test with 95\% confidence levels two-tailed (c, d, $\mathbf{f}$, $\mathbf{h}$ ). The gating strategy for $\mathbf{a}$ and $\mathbf{b}$ is shown in Supplementary Fig. 9a, gating strategy for $\mathbf{c}$ is shown in Supplementary Fig. 8a, gating strategy for $\mathbf{g}$ and $\mathbf{h}$ is shown in Supplementary Fig. 9 b.

\section{Methods}

Mice. C57BL/6 WT (C57BL/6JOlaHsd, Envigo, Israel), C57BL/6 (CD45.1), C57BL/ 6 SLAMF5-deficient ${ }^{29}$, JHT-deficient ${ }^{42}$, Vert-x (IL-10 GFP Reporter) ${ }^{45}$, and C57BL/6 SLAMF6-deficient ${ }^{63}$ mice were used at 6-12 weeks of age. Both females and males were used, and the groups where age- and sex-matched in each experiment, control mice were bred separately. All animal procedures were approved by the Animal Research Committee at the Weizmann Institute of Science. Mice were housed in an SPF animal facility, with an ambient temperature of $22 \pm 1^{\circ} \mathrm{C}$ in a light/dark cycle of $12 / 12 \mathrm{~h}$ with $50 \pm 5 \%$ humidity. Mice were euthanized using carbon dioxide $\left(\mathrm{CO}_{2}\right)$ overdose.

Experimental autoimmune encephalomyelitis Induction. Mice were injected subcutaneously with $200 \mu \mathrm{g} \mathrm{MOG}_{35-55}$, (synthesized by Genscript) in incomplete Freund's adjuvant supplemented with $3 \mathrm{mg} \mathrm{ml}^{-1}$ heat-inactivated Mycobacterium tuberculosis (Sigma-Aldrich). Pertussis toxin (Sigma-Aldrich), $200 \mathrm{ng}$ per mouse, was injected I.P. immediately after the $\mathrm{MOG}_{35-55}$ injection, and again after $48 \mathrm{~h}^{64}$.

Mice were examined daily and scored by a researcher blinded to the experimental treatment of the animals using the following scoring system: $0-$ no disease, 1-loss of tail tonicity, 2- hind leg weakness, 3-complete hind leg paralysis, 3.5-complete hind leg paralysis with partial hind body paralysis, 4-full hind and foreleg paralysis, and 5-moribund or dead animals ${ }^{64}$.

For EAE experiments, both males and females were used at ages 9-12 weeks, where age and sex-matched controls were used in each experiment.

Human peripheral blood lymphocyte preparation. Peripheral blood samples of MS patients and age- and sex-matched healthy controls were provided in compliance with the Institutional Review Board of the Barzilai Medical Center, Ashkelon, Israel. Consent was informed, and the samples were obtained by written consent. The Weizmann institute review board approved the study: IRB number 1339-1. Patient characteristics are listed in Supplementary Table 1. Peripheral blood samples of healthy controls were provided by the "Magen David Adom in Israel" blood bank. Peripheral blood mononuclear cells (PBMCs) homogenates were obtained by centrifuging the samples at $1400 \times g 5 \mathrm{~min}$ in order to separate the buffy coat, which was then treated with BD Pharm Lyse ${ }^{\mathrm{mw}}$ (BD Biosciences).

Mouse cell preparation. Mouse spleens and draining lymph nodes (LNs, including the axillary, brachial, inguinal, lumbar, and caudal LNs) were dissected postmortem and collected in PBS (Biological Industries) with $2 \%$ FCS. Briefly, cell homogenate was prepared, treated with red blood lysis buffer. Cells were then sashed and strained through $40-\mu \mathrm{m}$ nylon mesh and counted. Spinal cords were dissected postmortem into RPMI, by flushing the spinal cord out of the spine with RPMI (Gibco) using an 18-gauge needle. Due to the low lymphocyte yield from CNS tissue, four to five spinal cords were pooled for each measurement. Infiltrating leukocytes were prepared in a two separation gradients method; spinal cords were then gently homogenized using a glass tissue grinder in $5 \mathrm{ml}$ RPMI. Homogenate was added to a tube containing a $10 \mathrm{ml}$ RPMI, $9 \mathrm{ml}$ Percoll, and $1 \mathrm{ml} 10 \times$ PBS. Homogenate was then centrifuged at $7800 \times g$. for $30 \mathrm{~min}$ at RT. Leukocytes were then collected from the middle fraction of the gradient, strained through a $40-\mu \mathrm{m}$ nylon mesh, and washed with PBS. Leukocytes were then resuspended in $1 \mathrm{ml}$ buffer ( $1 \%$ bovine serum albumin, $0.02 \%$ sodium azide in calcium and magnesiumfree PBS) and underlay with $1 \mathrm{ml}$ ficoll. Samples were then centrifuged at $1400 \times \mathrm{g}$ for $25 \mathrm{~min}, \mathrm{RT}$, no brake. The Middle white layer, which contains the leukocytes, was then removed, washed, and counted ${ }^{65}$

Flow cytometry staining. Flow cytometry (FACS) analysis was performed using FACS Canto (BD Biosciences) and data were collected using FACSDIva8 (BD Biosciences). FACS data analysis was done using Flowjo v10. Antibodies are listed in Supplementary Table 2.

Intracellular cytokine analysis of T-cell cytokines and transcription was done following $24 \mathrm{~h}$ cultures of $5 \times 10^{6}$ cells per $\mathrm{ml}$ in $10 \%$ serum IMEM medium with $10 \mu \mathrm{g} \mathrm{ml}^{-1}$ MOG35-55. For the last $5 \mathrm{~h}$ of the culture PMA (100 $\mathrm{ng} \mathrm{ml}^{-1}$, SigmaAldrich), ionomycin $\left(1 \mu \mathrm{g} \mathrm{ml}^{-1}\right.$; Sigma-Aldrich), monensin, and brefeldin A (1:1000, Biolegend) were added.

For detection of IL-10 in B cells, B cells were cultured at $2.5 \times 10^{6}$ cells per $\mathrm{ml}$ in $10 \%$ serum IMEM with PMA (100 $\mathrm{ng} \mathrm{ml}^{-1}$, Sigma-Aldrich), ionomycin $\left(1 \mu \mathrm{g} \mathrm{ml}^{-1}\right.$; Sigma-Aldrich), and monensin (1:1000, Biolegend) for $5 \mathrm{~h}$ (use of PMA, ionomycin and monensin is abbreviated as PIM). Cells were then stained with surface markers followed by fixation, permeabilization, and incubation with intracellular Abs. For transcription factors, staining fixation was done using a transcription factor fixation/ permeabilization kit (Invitrogen), for cytokine staining fixation was done using BD Cytofix/Cytoperm (BD Biosciences).

Chimeric mice. CD45.1 C57BL/6 WT mice aged 6 weeks were lethally irradiated with 850 rad, X-ray (XRAD 320, PXI). After $24 \mathrm{~h}$, mice were injected with $2 \times 10^{6}$ BM cells consisting of $80 \%$ JHT-deficient BM and $20 \%$ WT or SLAMF5-deficient BM. After BM reconstitution (8-10 weeks), chimeric mice were induced with EAE using a milder protocol. Mice were injected with $150 \mu \mathrm{gOG}_{35-55}$ in incomplete Freund's adjuvant supplemented with $3 \mathrm{mg} \mathrm{ml}^{-1}$ heat-inactivated Mycobacterium tuberculosis (Sigma-Aldrich). Pertussis toxin, 50 ng per mouse, was injected I.P. immediately after the $\mathrm{MOG}_{35-55}$ injection, and again after $48 \mathrm{~h}$. For proliferation staining, B cells were treated with the cell proliferation dye (CPD) (eBiosceince) according to the manufacturer's instructions.

In vitro Breg generation assay. Spleens of naive mice were collected postmortem. $B$ cells were purified by positive B-cell selection with B220 magnetic beads (BD Biosciences). B cells were then incubated at a concentration of $2.5 \times 10^{6}$ per $\mathrm{ml}$ for 24 or $72 \mathrm{~h}$ in full IMEM with $10 \mu \mathrm{g} \mathrm{ml}^{-1}$ Lipopolysaccharide (LPS L2880, Sigma-Aldrich).

\section{SLAMF5 blocking-in vitro}

Mouse cells. B cells were isolated from WT, slamf $5^{-1-}$ or Vert-x mice in EAE remission or healthy naive WT, and incubated at a concentration of $2.5 \times 10^{6}$ cells per ml for 24 or $48 \mathrm{~h}$ in the presence of $5 \mu \mathrm{g} \mathrm{ml}^{-1}$ LPS (Sigma) and $30 \mu \mathrm{g} \mathrm{ml}^{-1}$ SLAMF5-blocking $\mathrm{Ab}^{30,31}$ or isotype control-IgG2a (MG2a-53, BioLegend), as previously described ${ }^{30,31}$

Human cells. B cells were isolated from PB derived from healthy donors by B cellnegative selection with EasySep ${ }^{m x}$ Human B Cell Isolation Kit (Stemcell Technologies). B cells were then incubated at a concentration of $2.5 \times 10^{6}$ per $\mathrm{ml}$ for 24 or $48 \mathrm{~h}$ in the presence of $30 \mu \mathrm{g} \mathrm{ml}^{-1}$ SLAMF5-blocking $\mathrm{Ab}(\mathrm{B} 4)^{30,31}$ or isotype control-IgG2a (BioLegend). 
Real-time reverse transcription-PCR analysis. RNA extraction was done using Direct-zol RNA microprep kit (Zymo Research). Reverse transcription was performed by Qiagen RT kit according to the manufacturer's instructions. qPCR was performed using a Light-Cycler 480 Instrument (Roche Diagnostics) and LightCycler HotStart DNA SYBR Green I mix kit (Roche Diagnostics). Primers used are listed in Supplementary Table 3.

RNA sequencing. For RNA preparation, $3 \times 10^{5} \mathrm{CD}^{+} 9^{+} \mathrm{GFP}^{+}$cells were sorted (using BD FACSMelody) from 2-3 EAE-induced Vert-x mice. Cells were incubated with SLAMF5-blocking or IgG-control Ab for $24 \mathrm{~h}$. Then the total RNA was extracted from the cells using the Direct-zol RNA kit (Zymo Research) according to the manufacturer's instructions. A bulk adaptation of the MARS-Seq protocol ${ }^{66}$ was utilized to generate the RNA-Seq libraries for expression profiling of both treatment groups. Briefly, RNA from each sample was barcoded during reverse transcription and then pooled. Subsequently, after cleanup using Agencourt Ampure XP beads (Beckman Coulter), the pooled samples were subjected to second-strand synthesis and were amplified linearly by T7 in vitro transcription. The resulting RNA was thereafter fragmented, tagged with Illumina sequences during ligation, and subjected to RT and PCR resulting in a sequencing-ready library. Libraries were quantified by Qubit, TapeStation, and qPCR, as previously described $^{66}$. Sequencing was done using a Nextseq 75 cycle high output kit (Illumina; paired-end sequencing).

Analysis of the MARS-seq was done using the UTAP pipeline (the Weizmann Institute Bioinformatics Unit) to map the reads to the mouse genome and to calculate Unique Molecule Identifier (UMI) counts per gene. Reads were trimmed using cutadapt (which removes adapter sequences from high throughput sequencing reads; with parameters: -a AGATCGGAAGAGCACACGTCTGAACT CCAGTCAC -a "A $\{10\}$ " -times $2-\mathrm{u} 3-\mathrm{u}-3-\mathrm{q} 20$-m 25). Reads were mapped to the (Mus_Musculus, UCSC, mm10) genome, STAR v2.4.2a. Genes having a minimum of five reads in at least one sample were considered for further analysis ${ }^{67}$. Differentially expressed gene (DEG) detection and count normalization analysis were performed by DESeq $2^{68} . P$ values in the UTAP results were adjusted for multiple testing using the Benjamini and Hochberg procedure; false discovery rate (FDR) correction was done by fdrtool ${ }^{69}$.

Breg T-cell suppression assay. T-cell suppression assay by regulatory B cells was performed ${ }^{70}$. Briefly, WT or slamf $5^{-1-}$ B cells were first purified by $\mathrm{B} 220^{+}$magnetic beads and then transitional 2 (T2) B cells were isolated by sorting from spleens of mice in remission from EAE, and cultured 1:1 or 2:1 with WT or slamf $55^{-1-} \mathrm{CD} 4{ }^{+} \mathrm{CD} 25^{-}$ $\mathrm{T}$ cells isolated by sorting for $72 \mathrm{~h}$ in the presence of $1 \mu \mathrm{g} \mathrm{ml}^{-1}$ anti-CD3 (17A2; Biolegend) and $3 \mathrm{mg} \mathrm{ml}^{-1}$ anti-CD28 (3751; Biolegend). To neutralize IL-10, cells were incubated with $10 \mu \mathrm{g} \mathrm{ml}^{-1}$ of mouse IL-10 antibody (MAB417, R\&D systems) or with IgG1-control (Biolegend).

Statistics. Statistical analysis was performed using GraphPad Prism 8 software (San Diego, California, USA). Disease scoring and weight loss graphs show s.e.m. The significance of disease score and weight loss was analyzed by calculating the area under the curve parameter (AUC). AUC analysis was performed using the Mann-Whitney test. Experiments analyzing peripheral leukocytes from EAEinduced mice included all mice with all scores. Experiments analyzing CNS leukocytes from EAE-induced mice included only mice of score $>=2$. When pooled samples were required (e.g., spinal cord analysis) all mice had the same score. The SLAMF5-blocking assay was analyzed using a two-tailed paired $t$ test; all other analyses were performed using one- or two-tailed unpaired $t$ test as appropriate. In the bar graphs, data represent mean \pm s.d., and each dot represents one biological repeat (mouse or human) unless specified otherwise.

Reporting summary. Further information on research design is available in the Nature Research Reporting Summary linked to this article.

\section{Data availability}

The RNA sequencing data discussed in this publication have been deposited in NCBI's Gene Expression Omnibus and are accessible through GEO Series accession number GSE160828. All other data are included in the Supplementary Information or available from the authors upon reasonable requests, as are unique reagents used in this Article. Source data are provided with this paper.

Received: 17 April 2020; Accepted: 1 March 2021; Published online: 25 March 2021

\section{References}

1. Filippi, M. et al. Multiple sclerosis. Nat. Rev. Dis. Prim. 4, 43 (2018).

2. Harp, C. T., Lovett-Racke, A. E., Racke, M. K., Frohman, E. M. \& Monson, N. L. Impact of myelin-specific antigen presenting B cells on $\mathrm{T}$ cell activation in multiple sclerosis. Clin. Immunol. 128, 382-391 (2008).
3. Elliott, C. et al. Functional identification of pathogenic autoantibody responses in patients with multiple sclerosis. Brain 135, 1819-1833 (2012).

4. Magliozzi, R. et al. Cerebrospinal fluid IgM levels in association with inflammatory pathways in multiple sclerosis patients. Front. Cell Neurosci. 14, 569827 (2020)

5. Villar, L. M. et al. Early differential diagnosis of multiple sclerosis using a new oligoclonal band test. Arch. Neurol. 62, 574-577 (2005)

6. Magraner, M. J. et al. Brain atrophy and lesion load are related to CSF lipidspecific IgM oligoclonal bands in clinically isolated syndromes. Neuroradiology 54, 5-12 (2012).

7. Alvord, E. C. J., Kies, M. W. \& Suckling, A. J. in Progress in Clinical and Biological Research. Vol. 146 (eds Alvord, E. C. Jr., Kies, M. W. \& Suckling, A. J.) 1-537 (Allen R. Liss, 1984).

8. Wekerle, H., Kojima, K., Lannes-Vieira, J., Lassmann, H. \& Linington, C. Animal models for multiple sclerosis. Ann. Neurol. 36, S47-S53 (1994).

9. Lyons, J. A., San, M., Happ, M. P. \& Cross, A. H. B cells are critical to induction of experimental allergic encephalomyelitis by protein but not by a short encephalitogenic peptide. Eur. J. Immunol. 29, 3432-3439 (1999).

10. Weber, M. S. et al. B-Cell activation influences t-cell polarization and outcome of anti-CD20 B-cell depletion in central nervous system autoimmunity. Ann. Neurol. 68, 369-383 (2010).

11. Lyons, J. A., Ramsbottom, M. J. \& Cross, A. H. Critical role of antigen-specific antibody in experimental autoimmune encephalomyelitis induced by recombinant myelin oligodendrocyte glycoprotein. Eur. J. Immunol. 32, 1905-1913 (2002).

12. Fernando, V. et al. Regulation of an autoimmune model for multiple sclerosis in Th2-biased GATA3 transgenic mice. Int. J. Mol. Sci. 15, 1700-1718 (2014).

13. Koutrolos, M., Berer, K., Kawakami, N., Wekerle, H. \& Krishnamoorthy, G. Treg cells mediate recovery from EAE by controlling effector $\mathrm{T}$ cell proliferation and motility in the CNS. Acta Neuropathol. Commun. 2, 163 (2014).

14. Vogelaar, C. F. et al. Fast direct neuronal signaling via the IL-4 receptor as therapeutic target in neuroinflammation. Sci. Transl. Med. 10, https://doi.org/ 10.1126/scitranslmed.aao2304 (2018).

15. Milo, R. Therapies for multiple sclerosis targeting B cells. Croat. Med. J. 60, 87-98 (2019)

16. Rosser, E. C. \& Mauri, C. Regulatory B cells: origin, phenotype, and function. Immunity 42, 607-612 (2015).

17. Floudas, A., Amu, S. \& Fallon, P. G. New insights into IL-10 dependent and IL-10 independent mechanisms of regulatory B cell immune suppression. J. Clin. Immunol. 36, 25-33 (2016).

18. Wolf, S. D., Dittel, B. N., Hardardottir, F. \& Janeway, C. A. Jr Experimental autoimmune encephalomyelitis induction in genetically B cell-deficient mice. J. Exp. Med. 184, 2271-2278 (1996).

19. Matsushita, T., Horikawa, M., Iwata, Y. \& Tedder, T. F. Regulatory B cells (B10 cells) and regulatory $\mathrm{T}$ cells have independent roles in controlling experimental autoimmune encephalomyelitis initiation and late-phase immunopathogenesis. J. Immunol. 185, 2240-2252 (2010).

20. Matsumoto, M. et al. Interleukin-10-producing plasmablasts exert regulatory function in autoimmune inflammation. Immunity 41, 1040-1051 (2014).

21. Fillatreau, S., Sweenie, C. H., McGeachy, M. J., Gray, D. \& Anderton, S. M. B cells regulate autoimmunity by provision of IL-10. Nat. Immunol. 3, 944-950 (2002).

22. Matsushita, T., Yanaba, K., Bouaziz, J. D., Fujimoto, M. \& Tedder, T. F. Regulatory B cells inhibit EAE initiation in mice while other B cells promote disease progression. J. Clin. Investig. 118, 3420-3430 (2008).

23. Ray, A., Basu, S., Williams, C. B., Salzman, N. H. \& Dittel, B. N. A novel IL-10independent regulatory role for B cells in suppressing autoimmunity by maintenance of regulatory T cells via GITR ligand. J. Immunol. 188, 3188-3198 (2012).

24. Flores-Borja, F. et al. CD19+CD24hiCD38hi B cells maintain regulatory $\mathrm{T}$ cells while limiting TH1 and TH17 differentiation. Sci. Transl. Med. 5 173 ra123 (2013)

25. Fouquet, G. et al. Signaling lymphocytic activation molecules Slam and cancers: friends or foes? Oncotarget 9, 16248-16262 (2018).

26. Martin, M. et al. CD84 functions as a homophilic adhesion molecule and enhances IFN-gamma secretion: adhesion is mediated by Ig-like domain $1 . J$. Immunol. 167, 3668-3676 (2001)

27. Romero, X. et al. CD229 (Ly9) lymphocyte cell surface receptor interacts homophilically through its $\mathrm{N}$-terminal domain and relocalizes to the immunological synapse. J. Immunol. 174, 7033-7042 (2005).

28. Yan, Q. R. et al. Structure of CD84 provides insight into SLAM family function. Proc. Natl Acad. Sci. USA 104, 10583-10588 (2007).

29. Cannons, J. L. et al. Optimal germinal center responses require a multistage $\mathrm{T}$ cell:B cell adhesion process involving integrins, SLAM-associated protein, and CD84. Immunity 32, 253-265 (2010).

30. Marom, A. et al. CD84 mediates CLL-microenvironment interactions. Oncogene 36, 628-638 (2017).

31. Binsky-Ehrenreich, I. et al. CD84 is a survival receptor for CLL cells. Oncogene 33, 1006-1016 (2014) 
32. Lewinsky, H. et al. CD84 regulates PD-1/PD-L1 expression and function in chronic lymphocytic leukemia. J. Clin. Invest 128, 5465-5478 (2018).

33. Gobel, K., Ruck, T. \& Meuth, S. G. Cytokine signaling in multiple sclerosis: lost in translation. Mult. Scler. 24, 432-439 (2018).

34. Aharoni, R. New findings and old controversies in the research of multiple sclerosis and its model experimental autoimmune encephalomyelitis. Expert Rev. Clin. Immunol. 9, 423-440 (2013).

35. Chastain, E. M., Duncan, D. S., Rodgers, J. M. \& Miller, S. D. The role of antigen presenting cells in multiple sclerosis. Biochim Biophys. Acta 1812, 265-274 (2011).

36. Pierson, E. R., Stromnes, I. M. \& Goverman, J. M. B cells promote induction of experimental autoimmune encephalomyelitis by facilitating reactivation of T cells in the central nervous system. J. Immunol. 192, 929-939 (2014).

37. Almolda, B., Gonzalez, B. \& Castellano, B. Antigen presentation in EAE: role of microglia, macrophages and dendritic cells. Front. Biosci. 16, 1157-1171 (2011).

38. Mauri, C. \& Bosma, A. Immune regulatory function of B cells. Annu. Rev. Immunol. 30, 221-241 (2012).

39. Mauri, C. \& Menon, M. The expanding family of regulatory B cells. Int. Immunol. 27, 479-486 (2015).

40. Ganti, S. N., Albershardt, T. C., Iritani, B. M. \& Ruddell, A. Regulatory B cells preferentially accumulate in tumor-draining lymph nodes and promote tumor growth. Sci. Rep. 5, 12255 (2015).

41. Shen, P. et al. IL-35-producing B cells are critical regulators of immunity during autoimmune and infectious diseases. Nature 507, 366-370 (2014)

42. Gu, H., Zou, Y. R. \& Rajewsky, K. Independent control of immunoglobulin switch recombination at individual switch regions evidenced through CreloxP-mediated gene targeting. Cell 73, 1155-1164 (1993).

43. Radomir, L. et al. T Cells regulate peripheral naive mature B cell survival by cell-cell contact mediated through SLAMF6 and SAP. J. Immunol. 199, 2745-2757 (2017).

44. Wang, N. et al. Negative regulation of humoral immunity due to interplay between the SLAMF1, SLAMF5, and SLAMF6 receptors. Front. Immunol. 6, 158 (2015).

45. Madan, R. et al. Nonredundant roles for B cell-derived IL-10 in immune counter-regulation. J. Immunol. 183, 2312-2320 (2009).

46. Tarique, $\mathrm{M}$. et al. Interleukin-10 producing regulatory $\mathrm{B}$ cells transformed $\mathrm{CD} 4(+) \mathrm{CD} 25(-)$ into Tregs and enhanced regulatory $\mathrm{T}$ cells function in human leprosy. Front. Immunol. 9, 1636 (2018).

47. Haining, W. N. \& Weiss, S. A. c-Maf in CD4(+) T cells: it's all about context. Nat. Immunol. 19, 429-431 (2018).

48. Liu, M. et al. Transcription factor c-Maf is essential for IL-10 gene expression in B cells. Scand. J. Immunol. 88, e12701 (2018).

49. Apetoh, L. et al. The aryl hydrocarbon receptor interacts with c-Maf to promote the differentiation of type 1 regulatory T cells induced by IL-27. Nat. Immunol. 11, 854-861 (2010).

50. Piper, C. J. M. et al. Aryl hydrocarbon receptor contributes to the transcriptional program of IL-10-producing regulatory B cells. Cell Rep. 29 , 1878-1892 e1877 (2019).

51. Vasileiadis, G. K. et al. Regulatory B and T lymphocytes in multiple sclerosis: friends or foes? Auto. Immun. Highlights 9, 9 (2018).

52. Knippenberg, S. et al. Reduction in IL-10 producing B cells (Breg) in multiple sclerosis is accompanied by a reduced naive/memory Breg ratio during a relapse but not in remission. J. Neuroimmunol. 239, 80-86 (2011).

53. Piancone, F. et al. B lymphocytes in multiple sclerosis: Bregs and BTLA/ CD272 expressing-CD19+ lymphocytes modulate disease severity. Sci. Rep. 6, 29699 (2016).

54. Thi Cuc, B., Pohar, J. \& Fillatreau, S. Understanding regulatory B cells in autoimmune diseases: the case of multiple sclerosis. Curr. Opin. Immunol. 61, 26-32 (2019).

55. Blair, P. A. et al. CD19(+)CD24(hi)CD38(hi) B cells exhibit regulatory capacity in healthy individuals but are functionally impaired in systemic Lupus Erythematosus patients. Immunity 32, 129-140 (2010).

56. Tedder, T. F. B10 cells: a functionally defined regulatory B cell subset. J. Immunol. 194, 1395-1401 (2015).

57. Mauri, C. \& Menon, M. Human regulatory B cells in health and disease: therapeutic potential. J. Clin. Investig. 127, 772-779 (2017).

58. Ray, A. \& Basu, S. Regulatory B cells in experimental autoimmune encephalomyelitis (EAE). Methods Mol. Biol. 1190, 243-255 (2014).

59. de Andres, C. et al. New regulatory CD19(+)CD25(+) B-cell subset in clinically isolated syndrome and multiple sclerosis relapse. Changes after glucocorticoids. J. Neuroimmunol. 270, 37-44 (2014),

60. Kinnunen, T. et al. Specific peripheral B cell tolerance defects in patients with multiple sclerosis. J. Clin. Investig. 123, 2737-2741 (2013).

61. Okada, Y. et al. Signaling via toll-like receptor 4 and CD40 in B cells plays a regulatory role in the pathogenesis of multiple sclerosis through interleukin-10 production. J. Autoimmun. 88, 103-113 (2018).
62. Staun-Ram, E. \& Miller, A. Effector and regulatory B cells in multiple sclerosis Clin. Immunol. 184, 11-25 (2017).

63. Zhao, F., Cannons, J. L., Dutta, M., Griffiths, G. M. \& Schwartzberg, P. L. Positive and negative signaling through SLAM receptors regulate synapse organization and thresholds of cytolysis. Immunity 36, 1003-1016 (2012).

64. Aharoni, R. et al. Immunomodulatory therapeutic effect of glatiramer acetate on several murine models of inflammatory bowel disease. J. Pharm. Exp. Ther 318, 68-78 (2006).

65. LaFrance-Corey, R. G. \& Howe, C. L. Isolation of brain-infiltrating leukocytes. J. Vis. Exp. https://doi.org/10.3791/2747 (2011).

66. Keren-Shaul, H. et al. MARS-seq2.0: an experimental and analytical pipeline for indexed sorting combined with single-cell RNA sequencing. Nat. Protoc. 14, 1841-1862 (2019).

67. Dobin, A. et al. STAR: ultrafast universal RNA-seq aligner. Bioinformatics $\mathbf{2 9}$, 15-21 (2013).

68. Love, M. I., Huber, W. \& Anders, S. Moderated estimation of fold change and dispersion for RNA-seq data with DESeq2. Genome Biol. 15, 550 (2014).

69. Strimmer, K. fdrtool: a versatile R package for estimating local and tail areabased false discovery rates. Bioinformatics 24, 1461-1462 (2008).

70. Rosser, E. C. \& Mauri, C. Regulatory B cells in experimental mouse models of arthritis. Methods Mol. Biol. 1190, 183-194 (2014).

\section{Acknowledgements}

The authors wish to thank members of the Shachar lab and Dr. Ziv Shulman for fruitful discussion and support. We would like to thank Dr. Chris Karp for providing the Vert-X mice. I.S. is the incumbent of Dr. Morton and Ann Kleiman Professorial Chair. This research was supported by ERA-NET TRANSCAN-2, Israel Science Foundation, and the Israel Cancer Research Foundation.

\section{Author contributions}

L.R.: designed the research, performed experiments, analyzed the data, and wrote the paper. M.P.: designed the research, performed experiments, and analyzed the data. N.S. designed the research, performed experiments, and analyzed the data. S.R.: performed experiments and analyzed the data. K.D.: performed experiments and analyzed the data A.W.: designed the research, performed experiments, and analyzed the data. M.P.K.: performed experiments and analyzed the data. H.L.: performed experiments and analyzed the data. S.B.-H.: performed experiments and analyzed the data. R.A.: designed the research and analyzed the data. R.M.: contributed vital reagent. C.M.: designed the research, analyzed the data, and wrote the paper. I.S.: designed research, analyzed the data, and wrote the paper.

\section{Competing interests}

The authors declare no competing interests.

\section{Additional information}

Supplementary information The online version contains supplementary material available at https://doi.org/10.1038/s41467-021-22230-z.

Correspondence and requests for materials should be addressed to I.S.

Peer review information Nature Communications thanks Bonnie Dittel, Manuel Friese and Luc Van Kaer for their contribution to the peer review of this work.

Reprints and permission information is available at http://www.nature.com/reprints

Publisher's note Springer Nature remains neutral with regard to jurisdictional claims in published maps and institutional affiliations.

\section{(i)}

Open Access This article is licensed under a Creative Commons Attribution 4.0 International License, which permits use, sharing, adaptation, distribution and reproduction in any medium or format, as long as you give appropriate credit to the original author(s) and the source, provide a link to the Creative Commons license, and indicate if changes were made. The images or other third party material in this article are included in the article's Creative Commons license, unless indicated otherwise in a credit line to the material. If material is not included in the article's Creative Commons license and your intended use is not permitted by statutory regulation or exceeds the permitted use, you will need to obtain permission directly from the copyright holder. To view a copy of this license, visit http://creativecommons.org/ licenses/by/4.0/.

(C) The Author(s) 2021 